\title{
NEW RESOLUTION STRATEGY FOR MULTI-SCALE REACTION WAVES USING TIME OPERATOR SPLITTING, SPACE ADAPTIVE MULTIRESOLUTION AND DEDICATED HIGH ORDER IMPLICIT/EXPLICIT TIME INTEGRATORS*
}

\author{
MAX DUARTE ${ }^{\dagger}$, MARC MASSOT $^{\dagger}$, STÉPHANE DESCOMBES $^{\S}$, CHRISTIAN TENAUD ${ }^{\uparrow}$, \\ THIERRY DUMONT", VIOLAINE LOUVET" , AND FRÉDÉRIQUE LAURENT ${ }^{\dagger}$
}

\begin{abstract}
We tackle the numerical simulation of reaction-diffusion equations modeling multiscale reaction waves. This type of problems induces peculiar difficulties and potentially large stiffness which stem from the broad spectrum of temporal scales in the nonlinear chemical source term as well as from the presence of steep spatial gradients in the reaction fronts, spatially very localized. In this paper, we introduce a new resolution strategy based on time operator splitting and space adaptive multiresolution in the context of very localized and stiff reaction fronts. It considers a high order implicit time integration of the reaction and an explicit one for the diffusion term in order to build a time operator splitting scheme that exploits efficiently the special features of each problem. Based on recent theoretical studies of numerical analysis such a strategy leads to a splitting time step which is not restricted neither by the fastest scales in the source term nor by stability constraints of the diffusive steps, but only by the physics of the phenomenon. We aim thus at solving complete models including all time and space scales within a prescribed accuracy, considering large simulation domains with conventional computing resources. The efficiency is evaluated through the numerical simulation of configurations which were so far, out of reach of standard methods in the field of nonlinear chemical dynamics for 2D spiral waves and 3D scroll waves as an illustration. Future extensions of the proposed strategy to more complex configurations involving other physical phenomena as well as optimization capability on new computer architectures are finally discussed.
\end{abstract}

Key words. Reaction-diffusion equations, multi-scale reaction waves, operator splitting, adaptive multiresolution

AMS subject classifications. 33K57, 35A18, 65M50, 65M08

1. Introduction. Numerical simulations of multi-scale phenomena are commonly used for modeling purposes in many applications such as combustion, chemical vapor deposition, or air pollution modeling. In general, all these models raise several difficulties created by the high number of unknowns, the wide range of temporal scales due to large and detailed chemical kinetic mechanisms, as well as steep spatial gradients associated with very localized fronts of high chemical activity. Furthermore, a natural stumbling block to perform 3D simulations with all scales resolution is either the unreasonably small time step due to stability requirements or the unreasonable memory requirements for implicit methods. In this context, one can consider various numerical strategies in order to treat the induced stiffness for time dependent

*This research was supported by a fundamental project grant from ANR (French National Research Agency - ANR Blancs) Séchelles (project leader S. Descombes - 2009-2013), by a CNRS PEPS Maths-ST2I project MIPAC (project leader V. Louvet - 2009-2010), and by a DIGITEO RTRA project MUSE (project leader M. Massot - 2010-2014).

${ }^{\dagger}$ Laboratoire EM2C - UPR CNRS 288, Ecole Centrale Paris, Grande Voie des Vignes, 92295 Châtenay-Malabry Cedex, France ( $\{\max$. duarte,marc.massot,frederique.laurent $\}$ @em2c.ecp.fr).

${ }^{\ddagger}$ Supported by a Ph.D. grant from Mathematics (INSMI) and Engineering (INSIS) Institutes of CNRS and by INCA project (CNRS/ONERA/SAFRAN).

$\S$ Laboratoire J. A. Dieudonné - UMR CNRS 6621, Université de Nice - Sophia Antipolis, Parc Valrose, 06108 Nice Cedex 02, France (sdescomb@unice.fr).

`LIMSI - CNRS, B.P. 133, Campus d'Orsay, 91403 Orsay Cedex, France (tenaud@limsi.fr).

"Institut Camille Jordan - UMR CNRS 5208, Université de Lyon, Université Lyon 1, INSA de Lyon 69621, Ecole Centrale de Lyon, 43 Boulevard du 11 novembre 1918, 69622 Villeurbanne Cedex, France ( $\{$ tdumont, louvet $\}$ @math . univ-lyon1.fr). 
problems. The most natural idea is to use dedicated numerical methods and to solve the complete models where diffusion, reaction and eventually convection are coupled together. One aims at solving strongly coupled nonlinear systems with either a fully implicit method, or yet semi-implicit or linearized implicit methods instead (see [9] and references therein). However, the strong stability restrictions for the latter when dealing with very fast temporal scales, as well as the computing cost and the high memory requirements of these methods, even if adaptive grids are used, make these strategies difficult to be handled.

An alternative numerical strategy is then to combine implicit and explicit schemes to discretize nonlinear evolution problems in time. Further studies settled the appropriate numerical background for these methods called IMEX, which in particular might be conceived to solve stiff nonlinear problems [35, 29]. These methods are usually very efficient but the feasibility of utilizing dedicated implicit solvers over a discretized domain becomes soon critical when treating large computational domains. On the other hand, the time steps globally imposed over partial regions or the entire domain are strongly limited by either the stability restrictions of the explicit solver or by the fastest scales treated by the implicit scheme. We know though that these fastest time scales do not always play a leading role in the global physics of many multi-scale problems and therefore one might consider the possibility of using reduced models where these chemical scales have been previously relaxed. These simplified models provide reasonable predictions and the associated computing costs are significantly reduced in comparison with comprehensive chemical models. Nevertheless, these reduced models provide only approximate solutions and are usually accessible when the system is well-partitioned and the fastest scales can be identified or isolated [30], a process that in realistic configurations, relies on sensitivity analysis which is most of the time difficult to conduct and justify.

It is then natural to envision a compromise, since the resolution of the fully coupled problem is most of the time out of reach and the appropriate definition of reduced models is usually difficult to establish. In this context, time operator splitting methods have been used for a long time and there exists a large literature showing the efficiency of such methods for evolution problems. A splitting procedure allows to consider dedicated solvers for the reaction part which is decoupled from the other physical phenomena like convection, diffusion or both, for which there also exist dedicated numerical methods. Hence, a completely independent optimization of the resolution of each subsystem might be pursued. In order to guarantee the accuracy of the solution obtained by a splitting scheme, the splitting time steps used for the independent resolution of each subproblem are usually taken of the order of the fastest scales included in the problem. As a matter of fact, several works [36, 31, 9] showed that the standard numerical analysis of splitting schemes fails in presence of scales much faster than the splitting time step. Nevertheless, more rigorous studies for these stiff configurations $[15,13]$ and in the case where spatial multi-scale phenomena arise as a consequence of steep spatial gradients [12], allow to characterize the behavior of splitting schemes with splitting time steps much larger than the fastest scales of the problem.

As a consequence, we introduce in this work a new time operator splitting approach for which the dedicated methods chosen for each subsystem are responsible for dealing with the fast scales associated with each one of them, in a separate manner. The global solution is then reconstructed by the splitting scheme with splitting time steps dictated by the global physical coupling, possibly much larger than the fastest 
time scales. In this way, the splitting time step is chosen based only on error estimates of the numerical simulation in order to guarantee the description of the physics of the phenomenon within a prescribed accuracy, without being related to the stability constraints of the numerical resolution of each subsystem and with an important improvement of efficiency whenever a broad decoupling of the time scale spectrum is possible.

The operator splitting strategy proposed in this article considers then a high order method like Radau5 [23], based on implicit Runge-Kutta schemes for stiff ODEs, to solve the reaction term; and on the other hand, another high order method like ROCK4 [1], based on explicit stabilized Runge-Kutta schemes, to solve the diffusion problem. In this way, the global accuracy of the time integration scheme is mainly set by the splitting scheme through the choice of the splitting time step. This numerical strategy is then complemented by a mesh refinement technique based on Harten's pioneering work on adaptive multiresolution methods [24], being aware of the interest of adaptive mesh techniques for problems exhibiting locally steep spatial gradients. Since a multiresolution technique allows to better control the accuracy of the adapted and compressed spatial representation, both the space and time errors can be regulated for a given semi-discretized problem. The main goal is then to perform computationally very efficient as well as accurate in time and space simulations of the complete dynamics of multi-scale phenomena under study with splitting time steps purely dictated by the physics of the phenomenon and not by any stability constraints associated with mesh size or source time scales. In particular, in the case of propagating wavefronts, we show that a constant splitting time step is good enough to capture the dynamics of the phenomenon even though an adaptive splitting time step technique as the one developed in [11] can be used to compute the appropriate splitting time step whenever the determination of the latter becomes difficult to conduct.

The paper is organized as follows. In section 2, we first recall the standard time operator splitting schemes. Then, we describe the new operator splitting strategy proposed for multi-scale problems, and its coupling with a suitable grid adaptation strategy, the space adaptive multiresolution technique [7, 26], which is briefly presented. The implementation of the numerical strategy is detailed in section 3 , as well as the algorithm scheme and the choice of the appropriate splitting time step. In section 4 , we present $2 \mathrm{D}$ and $3 \mathrm{D}$ simulations of a three species reaction-diffusion system modeling the Belousov-Zhabotinsky reaction, and we illustrate the potential and performance of the method by conducting 3D numerical simulations of very stiff reaction waves on a $512^{3}$ mesh size within a reasonable time on a workstation, a simulation out of reach of any standard method. We end in section 5 with some concluding remarks and prospects on future applications including other phenomena, and numerical developments on new parallel architecture where we can envision very large scale simulations.

2. Construction of the Numerical Strategy. In this section, we first recall some standard operator splitting schemes to then introduce a new splitting strategy for multi-scale waves modeled by stiff reaction-diffusion systems. In the last part, we detail briefly the adaptive multiresolution method that we have implemented as mesh refinement technique for this new resolution technique.

2.1. Time Operator Splitting. Let us first set the general mathematical framework of this work. A class of multi-scale phenomena can be modeled by general 
reaction-diffusion systems of type:

$$
\begin{aligned}
\partial_{t} \mathbf{u}-\partial_{\mathbf{x}}\left(\mathbf{D}(\mathbf{u}) \partial_{\mathbf{x}} \mathbf{u}\right) & =\mathbf{f}(\mathbf{u}), \quad \mathbf{x} \in \mathbb{R}^{d}, t>0 \\
\mathbf{u}(0, \mathbf{x}) & =\mathbf{u}_{0}(\mathbf{x}), \quad \mathbf{x} \in \mathbb{R}^{d}
\end{aligned}
$$

where $\mathbf{f}: \mathbb{R}^{m} \rightarrow \mathbb{R}^{m}$ and $\mathbf{u}: \mathbb{R} \times \mathbb{R}^{d} \rightarrow \mathbb{R}^{m}$, with the diffusion matrix $\mathbf{D}(\mathbf{u})$, which is a tensor of order $d \times d \times m$.

Even though the proposed numerical strategy handles general problem (2.1), in order to simplify the presentation we shall consider problem (2.1) with linear diagonal diffusion. In this case the elements of the diffusion matrix are written as $D_{i_{1} i_{2} i_{3}}(\mathbf{u})=$ $D_{i_{3}} \delta_{i_{1} i_{2}}$ for some positive indices $i_{1}, i_{2}, i_{3}$, so that the diffusion operator reduces to the heat operator with scalar diffusion coefficient $D_{i_{3}}$ for component $u_{i_{3}}$ of $\mathbf{u}$, $i_{3}=1, \ldots, m$. Performing a fine spatial discretization, we obtain the semi-discretized initial value problem

$$
\begin{aligned}
\mathrm{d}_{t} \mathbf{U}-\mathbf{B} \mathbf{U} & =\mathbf{F}(\mathbf{U}), \quad t>0, \\
\mathbf{U}(0) & =\mathbf{U}^{0},
\end{aligned}
$$

where $\mathbf{B}$ corresponds to the discretization of the Laplacian operator with the coefficients $D_{i_{3}}$ within. $\mathbf{U}$ and $\mathbf{F}(\mathbf{U})$ are arranged component-wise all over the discretized spatial domain. Considering a standard decoupling of the diffusion and reaction parts of (2.2), we denote $\mathcal{X}^{\Delta t}\left(\mathbf{U}^{0}\right)$ as the numerical solution of the diffusion equation

$$
\mathrm{d}_{t} \mathbf{U}_{D}-\mathbf{B} \mathbf{U}_{D}=0, \quad t>0
$$

with initial data $\mathbf{U}_{D}(0)=\mathbf{U}^{0}$ after an integration time step $\Delta t$. We also denote by $\mathcal{Y}^{\Delta t}\left(\mathbf{U}^{0}\right)$ the numerical solution of the reaction part,

$$
\mathrm{d}_{t} \mathbf{U}_{R}=\mathbf{F}\left(\mathbf{U}_{R}\right), \quad t>0,
$$

with initial data $\mathbf{U}_{R}(0)=\mathbf{U}^{0}$.

The two Lie approximation formulae of the solution of system (2.2) are then defined by

$$
\mathcal{L}_{1}^{\Delta t}\left(\mathbf{U}^{0}\right)=\mathcal{X}^{\Delta t} \mathcal{Y}^{\Delta t}\left(\mathbf{U}^{0}\right), \quad \mathcal{L}_{2}^{\Delta t}\left(\mathbf{U}^{0}\right)=\mathcal{Y}^{\Delta t} \mathcal{X}^{\Delta t}\left(\mathbf{U}^{0}\right)
$$

whereas the two Strang approximation formulae [32, 33] are given by

$$
\mathcal{S}_{1}^{\Delta t}\left(\mathbf{U}^{0}\right)=\mathcal{X}^{\Delta t / 2} \mathcal{Y}^{\Delta t} \mathcal{X}^{\Delta t / 2}\left(\mathbf{U}^{0}\right), \quad \mathcal{S}_{2}^{\Delta t}\left(\mathbf{U}^{0}\right)=\mathcal{Y}^{\Delta t / 2} \mathcal{X}^{\Delta t} \mathcal{Y}^{\Delta t / 2}\left(\mathbf{U}^{0}\right)
$$

where $\Delta t$ is now the splitting time step. It is well known that Lie formulae (2.5) (resp. Strang formulae (2.6)) are approximations of order 1 (resp. 2) of the exact solution of (2.2) in the case where $\mathcal{X}^{\Delta t}$ and $\mathcal{Y}^{\Delta t}$ are the exact solutions $X^{\Delta t}$ and $Y^{\Delta t}$ of problems (2.3) and (2.4). Then, appropriate numerical approximations of $X^{\Delta t}$ and $Y^{\Delta t}$ are required in order to compute Lie and Strang formulae with the prescribed order. Higher order splitting schemes are also possible. Nevertheless, the order conditions for such composition methods state that either negative time substeps or complex coefficients are necessary (see [22]). The last ones imply usually important stability restrictions and more sophisticated numerical implementations. In the particular case of negative time steps, they are completely undesirable for PDEs that are ill-posed for negative time progression. 
2.2. Time Integration Strategy. The standard orders achieved with a Lie or Strang scheme are no longer valid when we consider very stiff reactive or diffusive terms (see [15]). Furthermore, if the fastest time scales play a leading role in the global physics of the phenomenon, then the solution obtained by means of a operator splitting scheme will surely fail to capture the global dynamics of the phenomenon, unless we consider splitting time steps small enough to solve such scales.

In the opposite case when these fast scales are not directly related to the physical evolution of the phenomenon, larger splitting time steps might be considered, but order reductions may then appear due to short-life transients associated to fast variables. This is usually the case for propagating reaction waves where for instance, the speed of propagation is much slower than some of the chemical scales. In this context, it has been proved in [15] that better performances are expected while ending the splitting scheme by the time integration of the reaction part (2.4) or in a more general case, the part involving the fastest time scales of the phenomenon (see a numerical study with convection and complex chemistry in [13]). In particular, in the case of stiff reaction-diffusion systems with linear diagonal diffusion, no order loss is expected for the $\mathcal{L}_{2}^{\Delta t}$ and $\mathcal{S}_{2}^{\Delta t}$ schemes when fast scales are present in the reactive term. However, one must also take into account possible order reductions coming this time from space multi-scale phenomena due to steep spatial gradients whenever large splitting time steps are considered, as analyzed in [12].

These theoretical studies allow to depict more precisely the numerical behavior of the splitting techniques and thus, help us to select among the various splitting alternatives, depending on the nature of the problem. Nevertheless, the choice of suitable time integration methods for each subsystem is mandatory not only to guarantee such theoretical estimates but also to take advantage of the particular features of each independent subproblem in order to solve them with reasonable resources as accurately as possible. In particular, our splitting technique considers high order dedicated integration methods for each subproblem in order to properly solve the fastest time scales associated with each one of them, and in such a way that the main source of error is led by the operator splitting error. Then, the control of the accuracy of the time integration is ruled by the splitting scheme by means of the splitting time step needed to describe the global physical phenomenon within a required level of accuracy.

2.2.1. Time Integration of the Reaction: Radau5. Radau5 [23] is a fifth order implicit Runge-Kutta method for which order conditions proven by Butcher [6] are satisfied up to the order 5, whereas its stability function is generated by a collocation procedure with Radau quadrature formulae [18] that guarantees $A$ - and $L$-stability properties, so that very stiff systems of ODEs might be solved without any stability problem.

Nevertheless, nonlinear systems must be solved throughout the time integration process because of this implicit character. Even if the system resolution tools are highly optimized (which are based on modified Newton's methods), these procedures become very expensive for large systems and important memory requirements are needed in order to carry out these computations. As a consequence, the size of the system of equations to be solved is strongly limited by the computing resources. However, in a splitting scheme context, we easily overcome this difficulty because the reactive term of (2.2) is a system of ODEs without spatial coupling. Therefore, a local approach node by node is adopted where the memory requirements are only set by the number of local unknowns, which usually does not exceed conventional memory resources. Even more, this approach is posed as an embarrassingly parallel 
problem where no data exchange is needed among nodes, that therefore yields optimal load balancing on shared memory architectures (see for example the numerical implementations achieved in [17]).

A very important feature of the Radau5 solver is that precious computing time is saved because it considers an adaptive time stepping strategy which guarantees a requested accuracy of the numerical integration and at the same time, allows to discriminate stiff zones from regular ones so that small time steps are only considered for stiff behaviors. In a splitting context, the reaction time integration step $\Delta t_{R}$ will be adapted only at nodes where the reaction phenomenon takes place, yielding local reaction time steps much smaller than the splitting time step so that the global time advancement of the resolution given by the splitting time step $\Delta t$ will not be limited by these fast time scales. For multi-scale reaction waves, this adaptation happens in a very low percentage of the spatial domain, usually only in the neighborhood of the wavefront. On the other hand, larger time steps are considered at nodes with a chemistry at (partial) equilibrium. This local time stepping without data exchange nor reconstruction of intermediary values would not be possible if we integrated the entire reaction-diffusion system (2.2) at once.

2.2.2. Time Integration of the Diffusion: ROCK4. One of the most important advantages of ROCK4 [1] is its explicit character, hence the simplicity of its implementation. In fact, no sophisticated Linear Algebra tools are needed (no resolution of linear systems required) and thus, the resolution is based on simple matrix-vector products. Nevertheless, the computation cost relies directly on the requested quantity of such products, that is the number of internal stages $s$ needed within one time integration step of the diffusion problem, $\Delta t_{D}$, inside each splitting time step $\Delta t$. The memory requirements are also reduced as a consequence of its explicit scheme, nevertheless we must keep in mind that these requirements increase proportionally with the number of nodes considered over the spatial domain.

The ROCK solver [1] features also dynamic time step adaptation so that $\Delta t_{D}$ is chosen in order to guarantee a prescribed accuracy of computations. ROCK4 is formally a fourth order stabilized explicit Runge-Kutta method and such methods feature extended stability domain along the negative real axis [34]. Therefore, considering a general problem such as $\mathbf{v}^{\prime}=\mathbf{g}(\mathbf{v})$, in order to guarantee the stability of computations for a given time step $\Delta t_{D}$, the number of stages $s$ needed is directly related to the spectral radius $\rho(\partial \mathbf{g} / \partial \mathbf{v})$, as long as the latter is dominated by real negative eigenvalues. Hence, for a given $\Delta t_{D}$ needed to guarantee the accuracy of the integration, the minimum number of stages $s$ needed to guarantee the stability is computed by the ROCK4 solver through

$$
0.35 \cdot s^{2} \geq \Delta t_{D} \rho\left(\frac{\partial \mathbf{g}}{\partial \mathbf{v}}(\mathbf{v})\right)
$$

which extends quadratically on $s$ the stability domain of the method along the negative axis. Considering the construction of the ROCK4 scheme [1], at least $s=5$ internal stages are required.

The method is then very appropriate for diffusion problems because of the usual predominance of negative real eigenvalues for which the method is efficiently stable. A very suitable example is the linear diagonal diffusion problem (2.3) with only negative real eigenvalues and constant spectral radius $\rho(\mathbf{B})$. An important gain of efficiency is obtained in this case because the discretized diffusion operator has a sparse matrix 
structure that yields more performing matrix-vector products. In our particular applications, the diffusive phenomenon has a leading role of propagator of perturbations over the (partial) equilibrium nodes that result on excitation of the reactive schemes and thus, the propagation of the reaction wave. The resulting self-similar character implies that the number of stages needed will remain practically constant throughout the evolution of the phenomenon. The spectral radius must be previously estimated (for example, using the Gershgoring theorem or even numerically, as proposed by the ROCK4 solver by means of a nonlinear power method).

Once again, the implementation of this diffusion solver over the entire reactiondiffusion system (2.2) will not be appropriate under neither theoretical nor practical considerations, and highlights the inherited advantages of the time operator splitting. In particular, solving within a prescribed tolerance the diffusion problem may also yield $\Delta t_{D}$ much smaller than the splitting time step $\Delta t$, and thus, the global time advancement of the solution given by $\Delta t$ will not be necessarily limited by the diffusive time scales but by the global features of the coupled problem.

2.3. Mesh Refinement Technique. We are concerned with the propagation of reacting wavefronts, hence important reactive activity as well as steep spatial gradients are localized phenomena. This implies that if we consider the resolution of the reactive problem (2.4), a considerable amount of computing time is spent on nodes that are practically at (partial) equilibrium. We show for instance in [17] that for a numerical simulation with complex source mechanisms on a uniform grid, $60 \%$ of the computing time is spent on nodes with very reduced chemical activity. Moreover, there is no need to represent these quasi-stationary regions with the same spatial discretization needed to describe the reaction front, so that the diffusion problem (2.3) might also be solved over a smaller number of nodes. An adapted mesh obtained by a multiresolution process which discriminates the various space scales of the phenomenon, turns out to be a very convenient solution to overcome these difficulties; the basis of this strategy is presented in the following. For further details on adaptive multiresolution techniques, we refer to the books of Cohen [7] and Müller [26].

2.3.1. Basis of Multiresolution Representation. Let us consider nested finite volume discretizations of general problem (2.1) with only one component, $m=1$. For $j=0,1, \cdots, J$ from the coarsest to the finest grid, we build regular disjoint partitions (cells) $\left(\Omega_{\gamma}\right)_{\gamma \in S_{j}}$ of an open subset $\Omega \subset \mathbb{R}^{d}$, such that each $\Omega_{\gamma}, \gamma \in S_{j}$, is the union of a finite number of cells $\Omega_{\mu}, \mu \in S_{j+1}$, and thus, $S_{j}$ and $S_{j+1}$ are consecutive embedded grids. We denote $\mathbf{U}_{j}:=\left(u_{\gamma}\right)_{\gamma \in S_{j}}$ as the representation of $\mathbf{U}$ on the grid $S_{j}$ where $u_{\gamma}$ represents the cell-average of $u: \mathbb{R} \times \mathbb{R}^{d} \rightarrow \mathbb{R}$ in $\Omega_{\gamma}$,

$$
u_{\gamma}:=\left|\Omega_{\gamma}\right|^{-1} \int_{\Omega_{\gamma}} u(t, \mathbf{x}) \mathrm{d} \mathbf{x} .
$$

Data at different levels of discretization are related by two inter-level transformations which are defined as follows: (1), the projection operator $P_{j-1}^{j}$, which maps $\mathbf{U}_{j}$ to $\mathbf{U}_{j-1}$. It is obtained through exact averages computed at the finer level by

$$
u_{\gamma}=\left|\Omega_{\gamma}\right|^{-1} \sum_{|\mu|=|\gamma|+1, \Omega_{\mu} \subset \Omega_{\gamma}}\left|\Omega_{\mu}\right| u_{\mu}
$$

where $|\gamma|:=j$ if $\gamma \in S_{j}$. As far as grids are nested, this projection operator is exact and unique [7]. And (2), the prediction operator $P_{j}^{j-1}$, which maps $\mathbf{U}_{j-1}$ to an 
approximation $\hat{\mathbf{U}}_{j}$ of $\mathbf{U}_{j}$. There is an infinite number of choices to define $P_{j}^{j-1}$, but at least two basic constraints are usually imposed [8]:

1. The prediction is local, i.e., $\hat{u}_{\mu}$ depends on the values $u_{\gamma}$ on a finite stencil $R_{\mu}$ surrounding $\Omega_{\mu}$, where $|\mu|=|\gamma|+1$.

2. The prediction is consistent with the projection in the sense that

$$
\begin{aligned}
& \qquad u_{\gamma}=\left|\Omega_{\gamma}\right|^{-1} \sum_{|\mu|=|\gamma|+1, \Omega_{\mu} \subset \Omega_{\gamma}}\left|\Omega_{\mu}\right| \hat{u}_{\mu} ; \\
& \text { i.e., } P_{j-1}^{j} \circ P_{j}^{j-1}=I d .
\end{aligned}
$$

In the case where $P_{j}^{j-1}$ is linear, we have

$$
\hat{u}_{\mu}:=\sum_{\gamma} c_{\mu, \gamma} u_{\gamma}
$$

and if the prediction has some prescribed order $r>0$ of accuracy, then it is exact for polynomials of degree $r-1$, i.e., if $u \in \prod_{r-1}$, then $u_{\gamma}=\hat{u}_{\gamma}$ for all $\gamma[24,8]$.

With these operators, we define for each cell $\Omega_{\mu}$ the prediction error or detail as the difference between the exact and predicted values:

$$
d_{\mu}:=u_{\mu}-\hat{u}_{\mu},
$$

or in terms of inter-level operations:

$$
d_{\mu}=u_{\mu}-P_{|\mu|}^{|\mu|-1} \circ P_{|\mu|-1}^{|\mu|} u_{\mu} .
$$

The consistency assumption (2.10), the definitions of the projection operator (2.9) and of the detail (2.12) yield

$$
\sum_{|\mu|=|\gamma|+1, \Omega_{\mu} \subset \Omega_{\gamma}}\left|\Omega_{\mu}\right| d_{\mu}=0 .
$$

We can then construct as shown in [8] a detail vector defined as $\mathbf{D}_{j}=\left(d_{\mu}\right)_{\mu \in \nabla_{j}}$, where the set $\nabla_{j} \subset S_{j}$ is obtained by removing for each $\gamma \in S_{j-1}$ one $\mu \in S_{j}$ such that $\Omega_{\mu} \subset \Omega_{\gamma}$ in order to avoid redundancy from expressions (2.12) and (2.10), and to get a one-to-one correspondence:

$$
\mathbf{U}_{j} \longleftrightarrow\left(\mathbf{U}_{j-1}, \mathbf{D}_{j}\right)
$$

issued by operators $P_{j-1}^{j}$ and $P_{j}^{j-1}$. By iteration of this decomposition, we finally obtain a multi-scale representation of $\mathbf{U}_{J}$ in terms of $\mathbf{M}_{J}=\left(\mathbf{U}_{0}, \mathbf{D}_{1}, \mathbf{D}_{2}, \cdots, \mathbf{D}_{J}\right)$ :

$$
\mathcal{M}: \mathbf{U}_{J} \longmapsto \mathbf{M}_{J}
$$

2.3.2. Compression and Time Evolution on Graded Tree-structured Data. One of the main interests of carrying on such a multi-scale decomposition is that this new representation defines a whole set of regularity estimators all over the spatial domain and thus, a data compression might be achieved as follows.

Given a set of index $\Lambda \subset \nabla^{J}$ where $\nabla^{J}:=\bigcup_{j=0}^{J} \nabla_{j}$, we define a truncation operator $\mathcal{T}_{\Lambda}$, that leaves unchanged the component $d_{\lambda}$ if $\lambda \in \Lambda$ and replaces it by 0 , otherwise. In practice, we are interested in sets $\Lambda$ obtained by thresholding:

$$
\lambda \in \Lambda \text { if }\left|d_{\lambda}\right| \geq \varepsilon_{j}, \quad j=|\lambda|,
$$


with the level-dependent threshold values:

$$
\varepsilon_{j}=2^{\frac{d}{2}(j-J)} \varepsilon, \quad j=|\lambda|, \quad j \in[0, J],
$$

where $\varepsilon$ is the threshold value for the finest level $J$. A data compression is then obtained by discarding the cells whose details are not into $\Lambda$ according to (2.17), whereas the conservativity of the multiresolution scheme remains guaranteed by expression (2.14) as it can be shown in [26].

Nevertheless, allegedly useless details can not be deliberately deleted because a certain data structure must be respected in order to perform the different computations associated with the multi-scale transformation itself, mainly the prediction operator. The set $\Lambda$ must then exhibit a graded tree structure in order to guarantee the availability of cell values within the local prediction stencil (see [8, 28] for more details on the definition and construction of such structures). In this paper, we will not conduct the analysis of such data structures, but we present the following terminology related to a tree representation that we will adopt throughout this paper:

- If $\Omega_{\mu} \subset \Omega_{\lambda}$ with $|\mu|=|\lambda|+1$, we say that $\Omega_{\mu}$ is a child of $\Omega_{\gamma}$ and that $\Omega_{\gamma}$ is the parent of $\Omega_{\mu}$.

- Moreover, we define the leaves $\mathrm{L}(\Lambda)$ of a tree $\Lambda$ as the set of $\Omega_{\lambda}$ with $\lambda \in \mathrm{L}(\Lambda)$ such that $\Omega_{\lambda}$ has no children in $\Lambda$.

- Finally, we define $\Omega_{\lambda}$ as a root when it belongs to the coarsest grid, that is $\lambda \in S_{0}$ or $|\lambda|=0$, in which case, we denote $\lambda$ as $\lambda_{0}$.

An effective data compression is accomplished because $\mathbf{U}$ is not represented on the finest grid $S_{J}$ as $\mathbf{U}_{J}$ anymore, but on $\Lambda_{\varepsilon}$, where $\Lambda_{\varepsilon}$ is the smallest graded tree containing $\Lambda$ defined by (2.17). More precisely, the numerical solution $\mathbf{U}^{n}$ at time $n \Delta t$ can be represented on an adapted grid by the set $\left(u_{\lambda}^{n}\right)_{\lambda \in \mathrm{L}\left(\Lambda_{\varepsilon}^{n}\right)}$.

The time evolution is then performed only on the leaves of a fixed adapted grid. A refinement operator $\mathcal{R}$ is therefore defined in order to generate a set $\tilde{\Lambda}_{\varepsilon}^{n+1}$ containing $\Lambda_{\varepsilon}^{n}$, on which the time integration is computed, such that $\tilde{\Lambda}_{\varepsilon}^{n+1}$ is adapted for describing the solution at both $n \Delta t$ and $(n+1) \Delta t$. Operator $\mathcal{R}$ refines the adapted grid usually based on the values of the details, as in the classical Harten's refinement criteria [24]. The latter introduces for all $\lambda \in \mathrm{L}\left(\Lambda_{\varepsilon}^{n}\right)$ :

1. $\bar{k}$ cells in each direction at the same grid level $|\lambda|$ within the refined region if $\left|d_{\lambda}\right| \geq \varepsilon_{|\lambda|}$, in order to predict the propagation of the solution;

2. and furthermore, its $2^{d}$ children and thus, cells at the next grid level $|\lambda|+1$ if $\left|d_{\lambda}\right| \geq 2^{r} \varepsilon_{|\lambda|}$, where we recall that $r$ is the accuracy order of the prediction operator (2.11).

The added $u_{\lambda}^{n}$ with $\lambda \in \tilde{\Lambda}_{\varepsilon}^{n+1} \backslash \Lambda_{\varepsilon}^{n}$ can be constructed by applying the inverse operation of (2.16): $\mathcal{M}^{-1}$. The same refining procedure is adopted in our implementation but with a more conservative second criterion which adds the $2^{d}$ children of all $\lambda \in \mathrm{L}\left(\Lambda_{\varepsilon}^{n}\right)$ such that $\left|d_{\lambda}\right| \geq \varepsilon_{|\lambda|}$. In the practice, this adds one more grid level all over $\Lambda_{\varepsilon}^{n}$ which defines an often called security or safety layer (see e.g. $[28,5]$ ). On the other hand, Harten related the $\bar{k}$ cells in each direction to the support of the numerical flux evaluation and in the practice $\bar{k}=1$ was adopted since the time step is limited by a standard CFL condition [24]. In our numerical strategy, there is no such stability restriction on the splitting time step and the $\bar{k}$ cells are rather used to allow larger time evolution steps while ensuring an appropriate spatial representation as we will discuss in $\S 3.2$.

These refinement criteria are rather conservative, nevertheless they are simple and completely avoid unrefined resolution taking into account the choice of the time 
evolution steps detailed in $\S 3.2$ and the propagating nature of reaction waves at finite speed. The latter feature guarantees that a given adapted grid propagates along with the waves and no more that one grid level needs to be added at each time iteration. For more general cases, a more sophisticated refinement criteria might be required, as the one introduced in [8], that allow to add more than one refined level during one time step in order to properly describe new structures in the forthcoming solution. On the other hand, a dynamic time step adaptation strategy for highly unsteady problems such as the one developed in [16], shows that a high frequency of remeshing given by the adaptive time step allows also to rapidly capture the spatial dynamics of the problem within a prescribed tolerance of the adapted spatial representation.

An important theoretical result is that if we denote by $\mathbf{V}_{J}^{n}:=\left(v_{\lambda}^{n}\right)_{\lambda \in S_{J}}$, the solution fully computed on the finest grid, and denote by $\mathbf{U}_{J}^{n}$, the solution reconstructed on the finest grid that used adaptive multiresolution (keeping in mind that the time integration was really performed on the leaves $\mathrm{L}\left(\tilde{\Lambda}_{\varepsilon}^{n}\right)$ of a compressed representation of $\left.\mathbf{U}^{n}\right)$; then, for a fixed time $T=n \Delta t$, it can be shown that the error introduced by the compressed spatial representation is given by:

$$
\left\|\mathbf{U}_{J}^{n}-\mathbf{V}_{J}^{n}\right\| \propto n \varepsilon .
$$

This result was first stated by Harten in [24] for hyperbolic problems in a $L_{1}$-norm, where the multiresolution decomposition was used to accelerate the flux evaluations for a time integration performed on the finest uniform grid. Later on, (2.19) was mathematically proven in [8] under more rigorous constraints for the refinement criteria and the computation of the fluxes also for hyperbolic problems in a $L_{1}$-norm, this time for a fully multiresolution scheme where all operations were performed on the adapted grid. In the same spirit of these works, in this study we will consider (2.19) but with a $L^{2}$-norm, more suited for parabolic problems, as it was also previously considered for instance in $[28,5]$. Even though a fully mathematical proof is still required for this case, a numerical demonstration will be provided. Finally, the latest error estimate of the compressed spatial representation as well as the numerical behavior of the time operator splitting schemes, allow one to properly choose the simulation parameters in order to predict the expected level of accuracy of the resolution.

2.4. Summary of the Numerical Strategy. The resolution strategy can be summarized as follows:

$$
\begin{gathered}
\left(u_{\lambda}^{n}\right)_{\lambda \in \mathrm{L}\left(\tilde{\Lambda}_{\varepsilon}^{n}\right)} \stackrel{\mathcal{M}}{\longrightarrow}\left(u_{\lambda_{0}}^{n}, d_{\lambda}^{n}\right)_{\lambda \in \tilde{\Lambda}_{\varepsilon}^{n}} \\
\left(u_{\lambda_{0}}^{n}, d_{\lambda}^{n}\right)_{\lambda \in \tilde{\Lambda}_{\varepsilon}^{n}} \stackrel{\mathcal{T}_{\Lambda_{\varepsilon}^{n}}}{\longrightarrow}\left(u_{\lambda_{0}}^{n}, d_{\lambda}^{n}\right)_{\lambda \in \Lambda_{\varepsilon}^{n}} \\
\left(u_{\lambda_{0}}^{n}, d_{\lambda}^{n}\right)_{\lambda \in \Lambda_{\varepsilon}^{n}} \stackrel{\mathcal{R}}{\longrightarrow}\left(u_{\lambda_{0}}^{n}, d_{\lambda}^{n}\right)_{\lambda \in \tilde{\Lambda}_{\varepsilon}^{n+1}} \\
\left(u_{\lambda_{0}}^{n}, d_{\lambda}^{n}\right)_{\lambda \in \tilde{\Lambda}_{\varepsilon}^{n+1}} \stackrel{\mathcal{M}^{-1}}{\longrightarrow}\left(u_{\lambda}^{n}\right)_{\lambda \in \mathrm{L}\left(\tilde{\Lambda}_{\varepsilon}^{n+1}\right)} \\
\left(u_{\lambda}^{n}\right)_{\lambda \in \mathrm{L}\left(\tilde{\Lambda}_{\varepsilon}^{n+1}\right)} \stackrel{\mathcal{S}^{\Delta t}}{\longrightarrow}\left(u_{\lambda}^{n+1}\right)_{\lambda \in \mathrm{L}\left(\tilde{\Lambda}_{\varepsilon}^{n+1}\right)}
\end{gathered}
$$

The set $\left(u_{\lambda_{0}}^{n}\right)$ is defined as the set of roots of $\tilde{\Lambda}_{\varepsilon}^{n}$, that is all $\lambda \in \tilde{\Lambda}_{\varepsilon}^{n}$ such that $|\lambda|=0$ or $\lambda \in S_{0}$.

For $n=0$, the initial condition should be represented on $\mathrm{L}\left(\tilde{\Lambda}_{\varepsilon}^{0}\right)$ in step $(2.20)$, which can usually be the finest grid, that is all $\Omega_{\lambda}$ such that $|\lambda|=J$ or $\lambda \in S_{J}$. 
Nevertheless, this is not possible for large domains simulations, in which case, the initial condition is computed on an intermediate grid level $j$ : all $\Omega_{\lambda}$ such that $|\lambda|=j$ or $\lambda \in S_{j}$, then the solution is refined and recomputed over the next finer level after a thresholding process. This procedure is recursively applied until the pre-established finest level $J$ is reached; the general procedure is explained in details in [26].

The algorithm can schematically be represented as

$$
\mathbf{U}^{n+1}=\mathcal{S}^{\Delta t}\left(\mathcal{M}^{-1} \mathcal{R} \mathcal{T}_{\Lambda_{\varepsilon}^{n}} \mathcal{M} \mathbf{U}^{n}\right)
$$

with the compressed representations of $\mathbf{U}^{n+1}$ and $\mathbf{U}^{n}$ given by $\left(u_{\lambda}^{n+1}\right)_{\lambda \in \mathrm{L}\left(\tilde{\Lambda}_{\varepsilon}^{n+1}\right)}$ and $\left(u_{\lambda}^{n}\right)_{\lambda \in \mathrm{L}\left(\tilde{\Lambda}_{\varepsilon}^{n}\right)}$ respectively, and the Strang operator splitting $\mathcal{S}^{\Delta t}$ given by one of the formulae (2.6) as time evolution operator. One might add a last thresholding step to represent the solution on $\mathrm{L}\left(\Lambda_{\varepsilon}^{n+1}\right)$ instead of $\mathrm{L}\left(\tilde{\Lambda}_{\varepsilon}^{n+1}\right)$ in order to obtain slightly higher data compression. In what follows, the key aspects of the implemented algorithm are detailed.

3. Algorithm Implementation. A dynamic graded tree structure is used in this implementation to represent data in the computer memory. This kind of data structure has been used in other multiresolution applications [28] and other dedicated data structures have also been developed [3]. The adapted grid corresponds to a set of nested dyadic grids generated by refining recursively a given cell, depending on the local regularity of the solution. The chosen data structure can handle 1D, 2D and 3D Cartesian geometries, whereas the basic element of the structure is the cell itself, which consists of a set of geometric and physical values, plus pointers to its parent, their children and the contiguous cells in each dimension, the neighbors. Figure 3.1 shows an example of a graded tree structure in 1D.

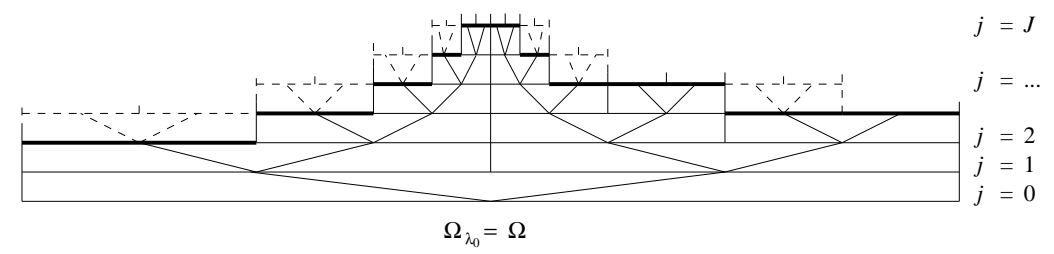

FIG. 3.1. Example of $1 D$ graded tree structure. Nodes and links to their corresponding children are indicated (solid lines) as well as the leaves (solid bold lines) and the phantoms (dashed lines).

The roots correspond to the basis of the tree, $\Omega_{\lambda_{0}}$, whereas the leaves are the upper elements with no children in the tree. In $d$ dimensions, a parent-cell at a level $j$ has at most $2^{d}$ children cells at level $j+1$. In this particular implementation, we impose that all $2^{d}$ children issued from the same parent-cell are simultaneously present if at least one child among them is needed. When there is only one root in the tree, the maximal number of leaves $N$ on which the solution might be represented is given by $N=2^{d J}$, which is exactly the number of cells on the finest grid. The maximal number of cells $M$ in the tree is given by $M=\left(2^{d(J+1)}-1\right) /\left(2^{d}-1\right)$. Additionally, although there is no rigorous mathematical analysis, it was shown in $[8,26]$ that a much more efficient alternative to fully evaluate the fluxes at the finest level considers the flux evaluations directly on the adapted mesh with essentially the same accuracy. Nevertheless, we consider in this work the intermediary case, also detailed in [26] and [28], that takes into account virtual cells called phantoms that are locally added to the tree in order to always compute the numerical fluxes of diffusion and convection operators at the 
highest grid level between two neighboring cells, following the procedure introduced in [28] for finite volume discretizations.

The implemented code represents the tree-structured data as a set of cells linked by pointers. In a FORTRAN 90/95 environment, a pointer is just an alias to the target; nevertheless, we take advantage of the fact that each pointer has a different state, depending on whether it is associated or not with another object. We work then with cells that are at different grids and that are not necessarily arranged in a contiguous way. Hence, we must conceive the mechanisms to navigate through the tree structure. In this implementation, we adopt a recursive strategy in which one moves from one cell to another passing by the child of the first, and by the consecutive children, until one gets to the desired cell. At each step, the state of the pointers tells us whether the target exists or not. In this recursive way, we are able to locate leaves or any cell and the same kind of procedure is conducted in the opposite direction, from leaves towards roots when necessary. Pointers to neighbors as well as other flags or indicators are not strictly necessary but eases considerably the searching process for certain routines.

3.1. Prediction Operator. To obtain the approximated values $\hat{u}_{\mu}$ by $P_{j}^{j-1}$ according to (2.11), we consider centered linear polynomial interpolations of order $2 l$, i.e. accuracy order $r=2 l+1$, computed with the $l$ nearest neighbors in each direction. For instance, in a $1 \mathrm{D}$ configuration for $l=1$, the prediction operator is explicitly given by:

$$
\begin{aligned}
\hat{u}_{j+1,2 k} & =u_{j, k}+\frac{1}{8}\left(u_{j, k-1}-u_{j, k+1}\right), \\
\hat{u}_{j+1,2 k+1} & =u_{j, k}+\frac{1}{8}\left(u_{j, k+1}-u_{j, k-1}\right),
\end{aligned}
$$

where the first index denotes the grid level and the second, the indexation of the cell into the tree. Higher order formula can be found in [26]. Since a Cartesian mesh is used, extension to multidimensional polynomial interpolations is easily obtained by a tensorial product of the 1D operator $[2,28]$. More accurate interpolations (higher $r$ ) yield better predicted values and thus, lower values of details following (2.12). Therefore, the thresholding process should also yield higher data compression according to (2.17). Nevertheless, this is not necessarily the case because larger stencils (higher $l$ ) and consequently more cells are needed to perform these interpolations so that the optimal choice of these parameters depends finally on the application. In the numerical illustrations we will restrict the different analysis to $l=1$, unless otherwise noted.

3.2. Choice of the Splitting Time Step. The splitting time step is set by the desired level of accuracy in the resolution of the wave speed, the wave profile, both, or any other parameter, depending on the problem and considering that each subsystem is perfectly solved. It is thus only depending on the global physics of the phenomenon we want to describe and therefore, on the degree of decoupling we can achieve between the various subsystems within a prescribed error tolerance. Considering the semidiscretized problem (2.2) with a sufficiently fine spatial discretization, if an accurate reference wave solution $\mathbf{U}$ or the corresponding wavefront speed $v$, can be computed either numerically or based on theoretical/analytical estimates, then the approximated solution $\mathbf{U}_{\text {split }}$ of speed $v_{\text {split }}$, computed with a splitting time step $\Delta t$ and an operator splitting technique with exact integration of the subsystems, must verify:

$$
E_{p}=\left\|\mathbf{U}-\mathbf{U}_{\text {split }}\right\|_{L^{2}} \leq \eta_{p}, \quad E_{v}=\left|v-v_{s p l i t}\right| / v \leq \eta_{v},
$$


where $\eta_{p}$ and $\eta_{v}$ are accuracy tolerances for the profile and velocity errors: $E_{p}$ and $E_{v}$, respectively. The profile error $E_{p}$ should be evaluated superposing both $\mathbf{U}$ and $\mathbf{U}_{\text {split }}$. However, a simpler and more practical strategy would just evaluate the $L^{2}$-error at a fixed time $t^{\star}$ :

$$
E=\left\|\mathbf{U}\left(t^{\star}\right)-\mathbf{U}_{\text {split }}\left(t^{\star}\right)\right\|_{L^{2}} \leq \eta
$$

in which case, both profile and velocity errors are simultaneously considered. For problems with more than one variable with different orders of magnitude, all these $L^{2}$-norms $\left(E_{p}\right.$ and $\left.E\right)$ that are independently performed for each variable, should be normalized by considering an appropriate scaling factor for the corresponding evaluated variable ${ }^{1}$. Notice that in order to remain coherent with the previous constraints and also to guarantee an accurate resolution of the reaction and diffusion problems, the corresponding accuracy tolerances $\eta_{\text {Radau } 5}$ and $\eta_{R O C K 4}$ of these solvers must verify:

$$
\eta_{\text {Radau } 5}, \eta_{R O C K 4}<\min \left\{\eta_{p}, \eta_{v}, \eta\right\}
$$

In this way, we can isolate the various integration errors and guarantee that the overall time integration error is practically given by the splitting scheme approximation. In particular, an evaluation of the sole splitting error allows to decouple the time scale spectrum of the problem whenever this is possible, contrary to classical applications of time operator splitting methods for which the splitting time step is directly settled by the fastest time scale of the phenomenon. In our strategy, we therefore extend the use of these methods to splitting time steps given by the global coupling scales, potentially larger than the fastest physical or numerical scales. This is a direct consequence of previous mathematical studies $[15,12]$ that demonstrate that even though there will possibly be a loss of order of these methods for time or space stiff problems and large splitting time steps, the splitting schemes will still consistently approximate the coupled resolution with an error piloted by the splitting time step.

We have established so far the criteria to handle time integration errors given by the splitting procedure. Nevertheless, the proposed strategy combines this splitting approach with a space multiresolution adaptive technique so that the approximation error introduced by the latter must be also taken into account. According to (2.19) and even though a rigorous mathematical demonstration is not yet available for parabolic problems, we consider as in previous studies $[28,5]$ the following error bound for a fixed time $T=n \Delta t$ :

$$
\left\|\mathbf{U}_{\text {split }}^{M R}-\mathbf{U}_{\text {split }}^{J}\right\|_{L^{2}} \leq C n \varepsilon
$$

for some positive $C$, where $\mathbf{U}_{\text {split }}^{M R}$ is the $\mathrm{MR} /$ splitting solution at $n \Delta t$ reconstructed on the finest grid $J$, which corresponds to the spatial discretization of the semidiscretized problem (2.2). A basic constraint to assume the validity of (3.5), as in the hyperbolic case, is that the propagating locally refined spatial gradients remain into the corresponding fine regions during each time step evolution $\Delta t$. In order to guarantee this for a given spatial discretization $(\Delta x, \Delta y, \Delta z)$ corresponding to the maximum $J$ level, the splitting time step must be bounded by a maximum splitting

\footnotetext{
${ }^{1}$ In the error evaluations conducted in this work, we have considered a scaling factor for each variable given by the corresponding maximum value of the variable.
} 
time step $\Delta t_{\max }$ computed by:

$$
\Delta t \leq \Delta t_{\max }=\min \left\{\frac{\hat{k} \Delta x}{v_{x}}, \frac{\hat{k} \Delta y}{v_{y}}, \frac{\hat{k} \Delta z}{v_{z}}\right\},
$$

for the directional components $\left(v_{x}, v_{y}, v_{z}\right)$ of the wavefront speed and where $\hat{k}=2+\bar{k}$ stands for the refined region in which the gradients might propagate without losing spatial resolution. The refined region is thus defined according to the refinement criteria detailed in $\S 2.3 .2$ for which one finer level is added everywhere $(2$ cells at $J$ in each direction since the corresponding $2^{d}$ children are simultaneously present in the safety layer) and $\bar{k}$ cells on the same level. We remark that the refined region $\hat{k}$ might be even larger in the practice because if one cell is a leaf, the remaining $2^{d}-1$ "brother-cells" issued from the same parent-cell are also leaves, even if some of them might not be necessary according to the threshold criterion (2.18).

We recall that there are no stability restrictions on the splitting time $\Delta t$ since the internal time integration solvers deal independently with these issues, and thus the splitting time step is uniquely associated with the numerical accuracy of the computations. In this context, the bound $\Delta t_{\max }$ is necessary only for adapted grids and allows simply to ensure an appropriate spatial representation of the steepest gradients and to decouple time and space approximation errors. That is, without this bound the propagating front might considerably leave the refined regions and the time integration performed on the resulting coarser cells might thus introduce complementary numerical errors into the numerical time integration process. Furthermore, the latter coupling of space and time features results usually into qualitatively different behaviors of the wave for instance in the velocity of propagation, which in the practice allows to identify an inappropriate choice of the splitting time step in the context of adapted grids. Finally, increasing the parameter $\bar{k}$ in the refinement criteria allows then to further enlarge the refined regions if larger splitting time steps are desired.

In the case of propagating wavefronts, a constant splitting time step based on a prescribed accuracy of the global time integration process as described in this section is more than reasonable, whereas the bound (3.6) guarantees a proper coupling of the space and time numerical methods. If no theoretical hints of the wave profile or velocity exist, the computation of a reference solution is usually very expensive but still feasible for one-dimensional or relatively small computational domains, which might give some insights in the behavior of the numerical methods in order to extrapolate these results to larger or multi-dimensional problems according to a standard numerical procedure. On the other hand, the speed of the wavefront needed to compute (3.6) can be always approximated by one-dimensional measurements taken either from fully coupled 1D configurations or for instance from feasible multi-dimensional $\mathrm{MR} /$ splitting resolutions for which the accuracy tolerances are tightened.

However, either if a more precise information on the accuracy of the time integration to choose the splitting time step is required, or if we are faced with highly unsteady problems, an adaptive splitting scheme introduced in [11] allows to properly estimate the local error of the splitting time integration without any need to compute a reference solution. Hence, based on these local error estimates, the splitting time steps are dynamically computed within a prescribed accuracy tolerance $\eta_{\Delta t}$. Nevertheless, this procedure introduces naturally an overhead which might not be justified in the simplified case of propagating waves so that a hybrid strategy that allows to consider a constant splitting time step issued from the local error estimates of the adaptive splitting procedure can be seen as the most convenient solution. In this 
work we are focused on the propagation of reaction waves, therefore the previously mentioned adaptive splitting scheme [11] is partially used only to justify and validate the chosen constant splitting time step and the proposed numerical strategy for this kind of problems.

3.3. Algorithm Scheme. The global algorithm can be summarized as: 1. INITIALIZATION:

- Initialization of parameters: e.g. maximum and minimum grid levels, domain size, number of roots.

- Initialization of the mesh structure:

- creation of the different grids;

- initialization of parameters of each cell from the roots, e.g. position, coordinates, level threshold value $\varepsilon_{j}$;

- definition of children and neighbors from the roots.

- Computation of initial solution at an intermediary grid level and recursive refinement and computation up to the maximum level.

2. LOOP IN TIME:

- Computation of cell values: projection operator $P_{j-1}^{j}$ from leaves towards roots.

- Computation of details: operator $\mathcal{M}$ from roots towards leaves.

- Thresholding and graduation: operator $\mathcal{T}_{\Lambda_{\varepsilon}}$ throughout the tree.

- Refinement of the tree: operator $\mathcal{R}$ throughout the tree.

- Computation of cell values from details: operator $\mathcal{M}^{-1}$ from roots towards leaves.

- Creation of phantom cells: needed for diffusion time step.

- Time integration: Strang operator splitting $\mathcal{S}_{2}^{\Delta t}$ applied only on leaves: - reaction half time step, time integration by Radau 5 cell by cell;

- diffusion time step, time integration by ROCK4 considering phantoms cells at grid level boundaries, computed by prediction operator;

- reaction half time step, time integration by Radau5 cell by cell.

\section{OUTPUT:}

Save adapted grid with the corresponding cell values represented on it.

Let us remark that during the thresholding process, cells are only labeled according to (2.17), but no cell is neither removed nor its detail set to zero, because a particular cell might be retained afterwards under the refinement or graduation criteria. On the other hand, the phantoms cells are created before the time integration process in order to separate the multiresolution and time integration operations in the code for this particular implementation. The values on the phantoms are nevertheless computed at each internal stage of ROCK4 by the prediction operator after locally updating their prediction stencils with the projection operator.

4. Numerical Simulations. In this last section, we present some numerical illustrations of the proposed strategy. A problem coming from nonlinear chemical dynamics is described and treated. The performance of the method is discussed in the context of $2 \mathrm{D}$ and $3 \mathrm{D}$ simulations. All simulations were performed on an AMDShanghai processor of $2.7 \mathrm{GHz}$ with memory capacity of $32 \mathrm{~GB}$.

4.1. Mathematical Model of Study. We are concerned with the numerical approximation of a model of the Belousov-Zhabotinski reaction, a catalyzed oxidation of an organic species by acid bromated ion (see [19] for more details and illustrations). We thus consider the model detailed in [21] and coming from the classic work of [20] 
which takes into account three species: hypobromous acid $\mathrm{HBrO}_{2}$, bromide ions $\mathrm{Br}^{-}$ and cerium(IV). Denoting by $a=[\mathrm{Ce}(\mathrm{IV})], b=\left[\mathrm{HBrO}_{2}\right]$ and $c=\left[\mathrm{Br}^{-}\right]$, we obtain a very stiff system of three PDEs:

$$
\begin{aligned}
\partial_{t} a-D_{a} \Delta a & =\frac{1}{\mu}(-q a-a b+f c), \\
\partial_{t} b-D_{b} \Delta b & =\frac{1}{\epsilon}(q a-a b+b(1-b)), \\
\partial_{t} c-D_{c} \Delta c & =b-c,
\end{aligned}
$$

with diffusion coefficients $D_{a}, D_{b}$ and $D_{c}$, and some real positive parameters $f$, small $q$, and small $\epsilon, \mu$, such that $\mu \ll \epsilon$.

The dynamical system associated with this system models reactive excitable media with a large time scale spectrum (see [21] for more details). Moreover, the spatial configuration with addition of diffusion generates propagating wavefronts with steep spatial gradients. Hence, this model presents all the difficulties associated with a stiff multi-scale configuration. The advantages of applying a splitting strategy to this problem have already been studied and presented in [14]. In what follows, we will consider 2D and 3D configurations of problem (4.1).

4.2. 2D BZ Equation. We first consider the 2D application of problem (4.1) with homogeneous Neumann boundary conditions and the following parameters, taken from [25] and a preliminary study [14]: $\epsilon=10^{-2}, \mu=10^{-5}, f=1.6$ and $q=2 \times 10^{-3}$, with diffusion coefficients $D_{a}=2.5 \times 10^{-3}, D_{b}=2.5 \times 10^{-3}$ and $D_{c}=1.5 \times 10^{-3}$. The phenomenon is studied over a time domain of $[0,4]$ and a space region of $[0,1]^{2}$. The initialization of the problem is based on [25] for the two-variable model of (4.1) with $b$ and $c$, and it is given by

$$
\begin{aligned}
a & =\frac{f c}{q+b}, \\
b & =0.8, \quad \text { if } 0<\theta<\arctan (0.3), \\
b & =b_{s s}, \quad \text { elsewhere }, \\
c & =c_{s s}+\frac{\theta}{8 \pi f},
\end{aligned}
$$

where $\theta$ is a polar coordinate angle considering as origin $(0.5,0.5)$ into $[0,1]^{2}$.

$$
b_{s s}=c_{s s}=q \frac{f+1}{f-1}
$$

is an approximation of the steady state values of the dynamical system associated with the two-variable problem obtained by taking (4.2) into the evolution equations of $b$ and $c$. (4.2) is obtained by taking $\mu \rightarrow 0$ into the evolution equation of $a$.

In the following, we will first consider a 2D computational domain with a uniform mesh of $256^{2}$ for which the coupled and split resolutions of the semi-discretized problem derived from (4.1) are rather expensive but still feasible. The idea is to compare these solutions with the ones computed by the proposed MR/splitting procedure in order to analyze the splitting and multiresolution errors regarding the corresponding accuracy tolerances detailed in $\S 3.2$ and to evaluate the computational performance of each approach. In a second step and based on these results we will study larger computational domains and 3D problems. 
We thus consider 8 nested dyadic grids with $N=2^{2 \times 8}=65536=256^{2}$ cells on the finest grid $J=8$ and define a reference or quasi-exact solution $\mathbf{U}_{q e}^{J}$ as the solution of the semi-discretized coupled reaction-diffusion problem (4.1) on the finest mesh $J$ performed by ROCK4 with very fine tolerances, $\eta_{R O C K 4}=10^{-14}$. For this problem and with the previous parameters, the spectral radius of the Jacobian of the reaction term into (4.1) is usually dominated by the negative real parts of the associated eigenvalues even though the imaginary parts are also present. Therefore only fine tolerances that yield sufficiently small time steps allow to fully guarantee the stability of the ROCK4 scheme whenever the imaginary part appears, considering the reduced stability domains of these methods along the imaginary axis. The main limitation to directly perform such computation with the Radau5 solver comes from its important memory requirements.

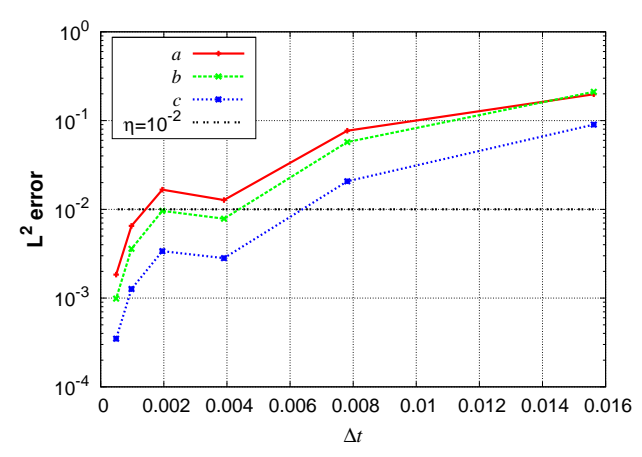

FIG. 4.1. 2D BZ spiral waves. Normalized $L^{2}$-errors for several splitting time steps $\Delta t$ at final time $t^{\star}=4$ according to (3.3) and prefixed accuracy tolerance of $\eta=10^{-2}$. Uniform grid of $256^{2}$.

The split solution $\mathbf{U}_{\text {split }}^{J}$ uses the RDR Strang $\mathcal{S}_{2}^{\Delta t}$ scheme as time integration method of the semi-discretized problem (4.1) with Radau5 for the time integration of the reaction term and ROCK4 for the diffusive part, $\eta_{\text {Radau } 5}=\eta_{R O C K 4}=10^{-5}$. In order to choose the appropriate splitting time step $\Delta t$, we set an accuracy tolerance of $\eta=10^{-2}$ considering for simplicity the normalized $L^{2}$-errors (3.3) as explained in $\S 3.2$. Figure 4.1 shows these errors evaluated at final time $t^{\star}=4$ for all three variables. A rounded value of splitting time step of $\Delta t=4 / 1024 \approx 3.91 \times 10^{-3}$ is finally chosen for which $L^{2}$-errors are close to $\eta$ for all three variables and times $t^{\star} \in[0,4]$ into (3.3). In this work, we have computed several split solutions with different time steps for the whole time domain in order to analyze the different aspects of the scheme. Nevertheless, in a practical situation and for the simulation of propagating waves, we can consider a much less expensive procedure that evaluates the local errors after one splitting time step starting from an intermediary solution for which the waves are fully developed.

A rather large accuracy tolerance $\eta$ was considered in order to show the decoupling of time steps for reaction, diffusion and the time operator splitting needed to solve the problem within the prescribed tolerance. The imposed tolerances for the reaction resolution imply time steps varying from $8.88 \times 10^{-5}$ to $\Delta t / 2 \approx 1.95 \times 10^{-3}$ for points located respectively in the neighborhood of the reactive front and the reduced chemical activity regions. On the other hand, the selected tolerances for ROCK4 yield time steps $\Delta t_{D}$ relatively constant of about $6.5-8 \times 10^{-4}$, that is $5-6$ diffusion time steps within each splitting time step $\Delta t$. For the spatial discretization of $256^{2}$, the spectral radius $\rho(\mathbf{B})$ estimated by ROCK4 is about 1400 , so that no more than the 
minimum number of stages $s=5$ is required according to (2.7). As a consequence, a CPU time of $1029 \mathrm{~s}$ is needed compared with the coupled resolution with ROCK4 that takes $23967 \mathrm{~s}$. The latter considers time steps of about $2.4 \times 10^{-6}$ given by the fine tolerances and 5 internal stages for a larger spectral radius of 95000 , that considers both reaction- and diffusion-associated eigenvalues. Even though this coupled resolution should be more accurate than a splitting technique, it will be no longer feasible for larger computational domains and moreover not appropriate for more complex chemical terms ${ }^{2}$.
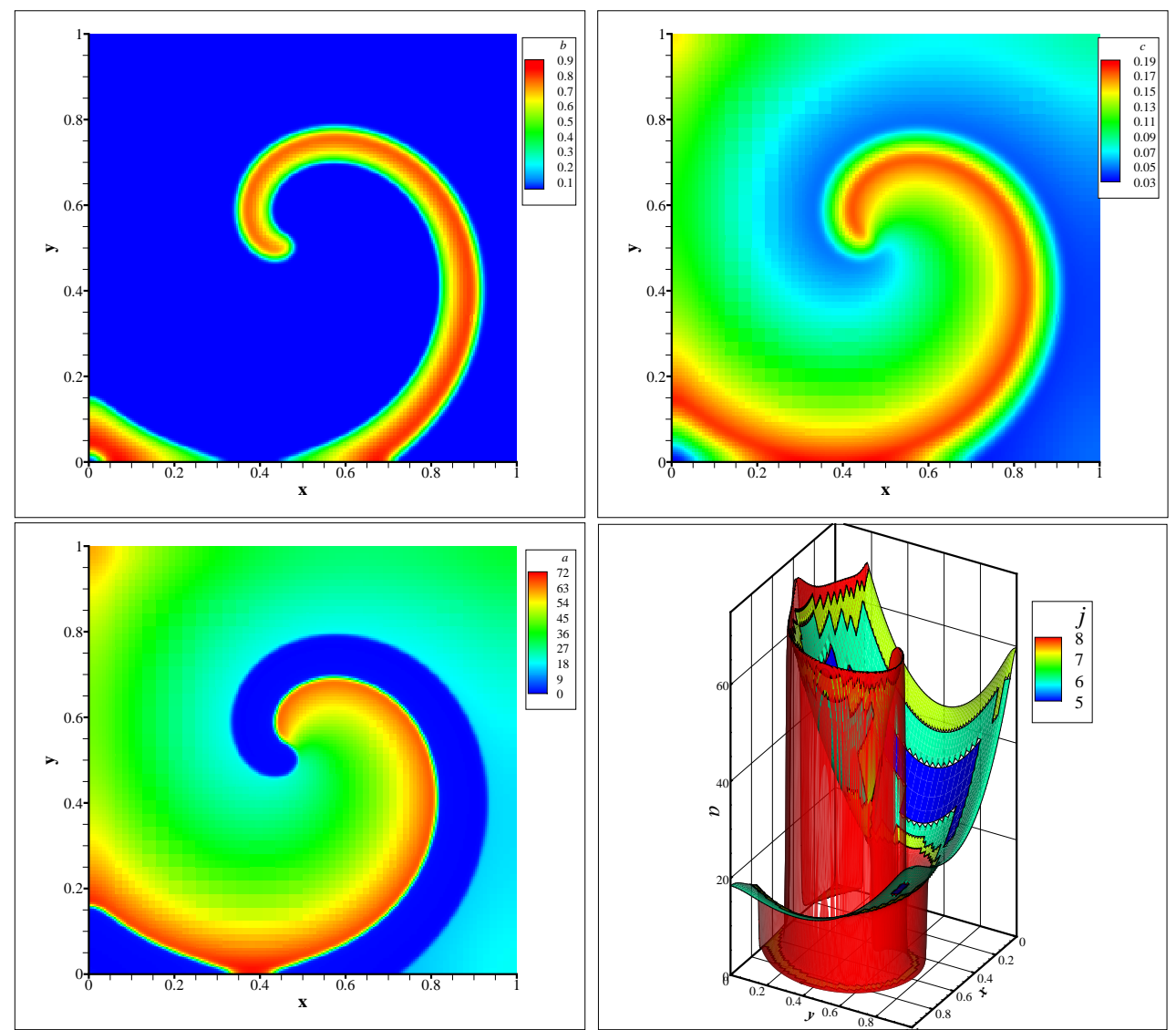

FIG. 4.2. 2D BZ spiral waves. Top: variable b (left) and c (right) at $t=4$. Bottom: variable $a$ (left) and its representation with four levels of mesh discretization with $\varepsilon=10^{-2}$ (right). Finest grid: $256^{2}$.

We consider now the proposed strategy that combines the previous splitting solver with the multiresolution adaptive technique. Figure 4.2 shows the spiral waves and the four different levels of spatial discretization on which they have been simulated with $\varepsilon=10^{-2}$ for the stiffest variable $a$. Whenever we consider grid adaptation the bound (3.6) on the splitting time step $\Delta t$ must be taken into account. We need then to estimate the speed of propagation $v$ of the wavefront. This can be done by computing the propagating speed of each variable along each direction as it is shown

\footnotetext{
${ }^{2}$ For instance, for the coupled problem (4.1) ROCK4 starts showing stability problems for $\eta_{R O C K 4}<10^{-5}$.
} 
in Figure 4.3 for variable $a$ along the $y$-axis. For the BZ waves, we have estimated a maximum speed of $v_{x}=v_{y} \approx 0.7$, which yields a maximum splitting time step $\Delta t_{\max }=1.6 \times 10^{-2}$ for $\Delta x=\Delta y=1 / 256$ and $\hat{k}=3$, considering one enlarging cell in each direction $\bar{k}=1$ in the refinement criteria. In this particular case, we can also compute the speed relative error $E_{v}$ following (3.2) between the quasi-exact and a splitting solution with $\Delta t=4 / 1024$. These errors remain practically lower than $0.2 \%$ as seen in Figure 4.3, which imply an accuracy tolerance of $\eta_{v}=2 \times 10^{-3}$ considering the speed resolution.
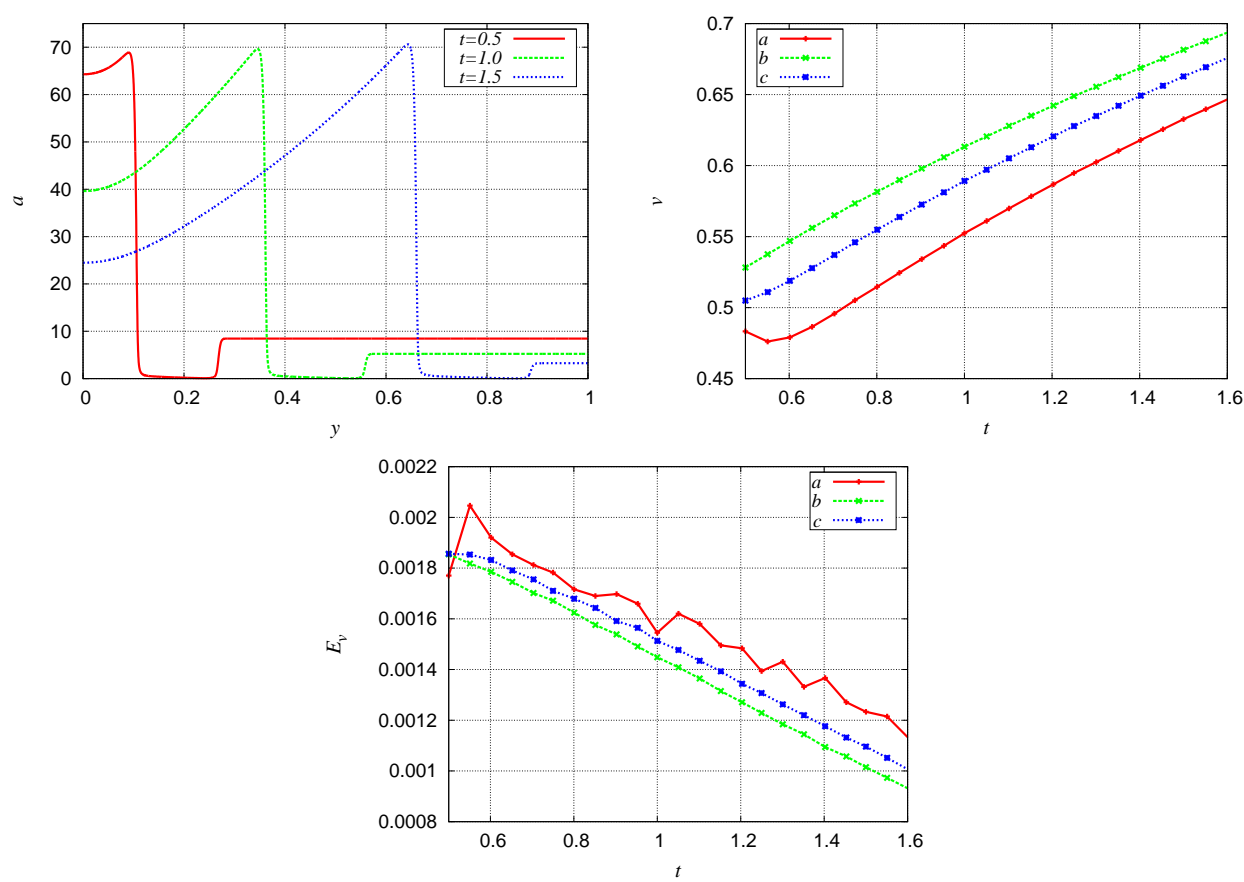

FIG. 4.3. BZ wave speed for splitting time step $\Delta t=4 / 1024$. Top left: time evolution of variable a along y-axis (see Figure 4.2); and right, estimated wavefront speed v. Bottom: speed relative errors $E_{v}$ for all three variables according to (3.2).

TABLE 4.1

2D BZ. CPU time in seconds for quasi-exact, splitting and MR/splitting solutions with different threshold values $\varepsilon$. Finest grid: $256^{2}$.

\begin{tabular}{|c|c|c|c|c|c|c|}
\hline \hline MR/splitting $\varepsilon=$ & $10^{-1}$ & $10^{-2}$ & $10^{-3}$ & $10^{-4}$ & splitting & quasi-exact \\
\hline \hline CPU time (s) & 536 & 886 & 1233 & 2402 & 1029 & 23967 \\
\hline \hline
\end{tabular}

The proposed MR/splitting strategy represents and computes solutions only on adapted grids, the leaves of the tree structure, throughout the time domain. Therefore, we define the data compression $(D C)$ as one minus the ratio between the number of cells on the adapted grid $(A G)$ and those on the finest uniform grid $(F G)$, expressing the whole as a percentage:

$$
D C=\left(1-\frac{A G}{F G}\right) \times 100
$$


Figure 4.4 shows different data compression rates for several threshold values. Smaller threshold values $\varepsilon$ imply more refinement and thus, compressions are less important. The whole finest grid is necessary for $\varepsilon<10^{-5}$. The corresponding CPU times for each one of these computations are included in Table (4.1) along with the quasi-exact and the splitting solution without any grid adaptation.

A more precise analysis of the CPU time consumption summarized in Table (4.2) shows that, as it was expected, an adapted grid allows to significantly reduce the time cost of the reaction integration as a consequence of the important reduction of the number of points without any chemical activity. On the other hand even though for $\varepsilon=10^{-2}$ we consider only $25 \%$ of the $256^{2}$ points, an important overhead is introduced in the time integration of the diffusion because the introduction of phantoms cells yield denser matrix representations of the discretized diffusion operator and thus more expensive matrix-vector products. For these stiff problems, the MR operations represent less than $15 \%$ of the total time, whereas the construction of the diffusion matrix takes over $6 \%$ since it has to be recomputed at each splitting time step contrary to a uniform grid representation for which this matrix is constant. From a practical point of view, we can see that a more efficient strategy will directly consider the adaptive grid for the flux evaluations as detailed in [26] without the introduction of any phantoms cells. Nevertheless, in this work we will keep the previous approach for being more accurate and taking into account that better performances are expected for larger computational domains for which grid adaptation is mandatory for the feasibility of simulations. An overhead is introduced in the CPU times in Table (4.2) coming from the profiling of the codes.

TABLE 4.2

2D BZ. CPU time in seconds for the reaction and diffusion time integrations for a splitting and a $M R /$ splitting resolution with $\varepsilon=10^{-2}$. Finest grid: $256^{2}$.

\begin{tabular}{|c|c|c|c|c|}
\hline \hline & \multicolumn{2}{|c|}{ splitting } & \multicolumn{2}{c|}{ MR/splitting $\varepsilon=10^{-2}$} \\
& CPU time (s) & $\%$ & CPU time (s) & $\%$ \\
\hline \hline Reaction & 963 & 65.4 & 486 & 44.0 \\
Diffusion & 481 & 32.7 & 348 & 31.5 \\
\hline \hline Total & 1472 & 100.0 & 1104 & 100.0 \\
\hline \hline
\end{tabular}

We consider now the numerical accuracy of the MR/splitting strategy $\mathbf{U}_{\text {split }}^{M R}$, discussed in $\S 3.2$, with respect to the reference solution, $\mathbf{U}_{q e}^{J}$, for the semi-discretized problem (4.1) on a uniform mesh $J$ given by $256^{2}$ points. The accuracy of the splitting scheme $\mathbf{U}_{\text {split }}^{J}$ applied to (4.1) discretized on the same uniform mesh $J$, is given by an accuracy tolerance $\eta$ according to (3.3) through the proper choice of the splitting time step $\Delta t$ (see Figure 4.1) regardless the possible loss of order for the Strang $\mathcal{S}_{2}^{\Delta t}$ scheme [15, 12]. On the other hand, a multiresolution decomposition yields a compressed spatial representation whose accuracy to approximate the corresponding uniform mesh representation is related to the threshold value $\varepsilon$ through (3.5). At some fixed time $t^{\star}$ the overall numerical accuracy of the $\mathrm{MR} /$ splitting resolution is then set by the previous splitting and multiresolution errors:

$$
\left\|\mathbf{U}_{q e}^{J}-\mathbf{U}_{\text {split }}^{M R}\right\|_{L^{2}} \leq\left\|\mathbf{U}_{q e}^{J}-\mathbf{U}_{\text {split }}^{J}\right\|_{L^{2}}+\left\|\mathbf{U}_{\text {split }}^{J}-\mathbf{U}_{\text {split }}^{M R}\right\|_{L^{2}} .
$$

Figure 4.4 shows the corresponding normalized $L^{2}$ errors at $t^{\star}=4$ for several threshold values $\varepsilon$ and $\eta=10^{-2}$ for a splitting time step of $\Delta t=4 / 1024$. Multiresolution errors are evaluated at the finest grid $J$ after reconstruction from the adapted mesh solution 
and depend proportionally on the imposed threshold value $\varepsilon$ according to (3.5). For this time multi-scale phenomenon, the accuracy of the MR/splitting strategy should be fixed by the time integration process in order to guarantee an appropriate resolution of the time scale spectrum of the stiff problem, whereas the multiresolution procedure allows to compress the spatial representation by retaining the desired level of refinement only wherever it is necessary, taking into account the space multi-scale features of the physical problem. In this case, these error estimates show that for $\varepsilon \leq 10^{-2}$, the multiresolution errors become negligible compared with the operator splitting ones so that the overall accuracy is indeed given by $\eta$.
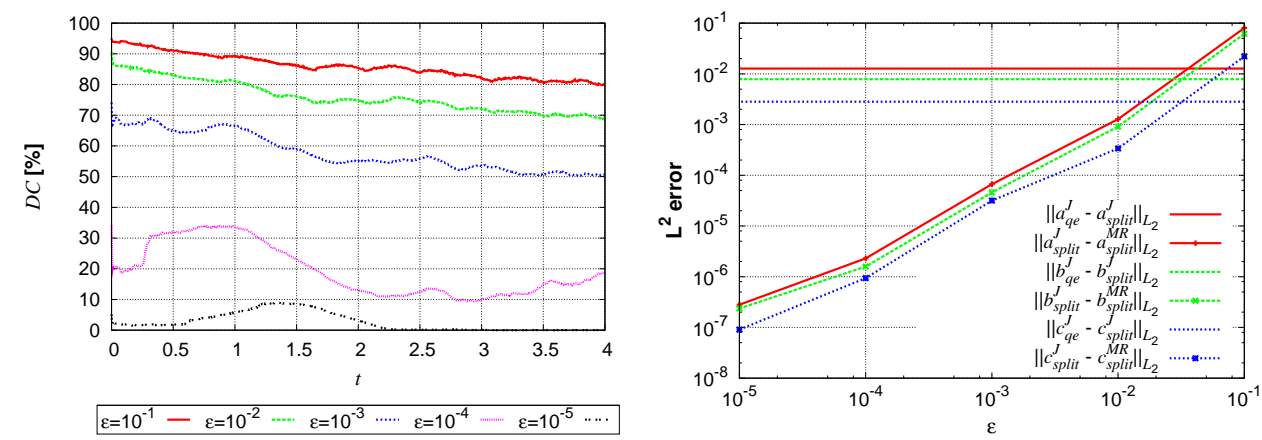

FIG. 4.4. 2D BZ spiral waves. Left: time evolution of the data compression DC given by (4.6) in percentage. Right: normalized $L^{2}$ errors at $t^{\star}=4$ given by the splitting technique on a uniform grid according to (3.3), and by the MR procedure according to (3.5), for several threshold values $\varepsilon$ and splitting time step of $\Delta t=4 / 1024$. Finest grid: $256^{2}$.
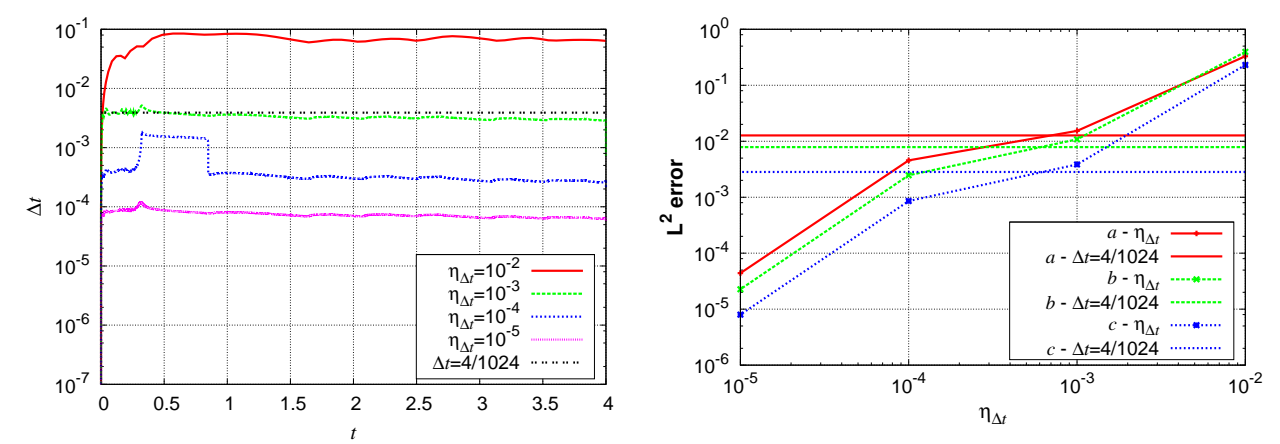

FIG. 4.5. 2D BZ spiral waves. Time evolution of splitting time steps (left) and normalized $L^{2}$ errors at $t^{\star}=4$ (right) given by the splitting technique on a uniform grid of $256^{2}$ according to (3.3) for different accuracy tolerances $\eta_{\Delta t}$ for the time adaptive strategy [11] and with $\Delta t=4 / 1024$.

Figure 4.5 shows the adaptive splitting time steps corresponding to different accuracy tolerances $\eta_{\Delta t}$ for the local error of the splitting time integration, according to the time adaptive procedure introduced in [11]. In all cases, the splitting time step is adapted from a chosen initial value of $\Delta t=10^{-7}$ to a roughly constant value that depends on the prescribed accuracy due to the self-similar character of the wave. The global error is indeed controlled by the local error accuracy tolerance $\eta_{\Delta t}$ as it is shown by the normalized $L^{2}$ errors at $t^{\star}=4$ according to (3.3). We see thus that for this kind of propagating phenomena a constant splitting time step computed based 
on an accuracy criterion as detailed in $\S 3.2$, is appropriate to describe accurately the multi-scale phenomenon. Furthermore, a splitting time step of $\Delta t=4 / 1024$ yields practically the same results as the adaptive splitting strategy with $\eta_{\Delta t}=10^{-3}$, so that the overhead of estimating the local errors of the adaptive scheme can be saved, even though this overhead implies no more than $25 \%$ of additional CPU time considering the embedded procedure developed in [11]. Just like before, the same time integration and thus global errors are found for $\eta_{\Delta t}=10^{-3}$ and different adapted meshes as long as $\varepsilon \leq 10^{-2}$.

We have analyzed so far the numerical behavior of the proposed numerical strategy in terms of the splitting time integration method, the computational costs and the numerical errors for a computational domain of $256^{2}$ points, that allows to represents accurately enough the multi-scale phenomenon and furthermore, it allows to conduct several computations with reasonable computational resources. Let us now consider a more challenging configuration with larger computational domain in order to complete the present study. We therefore consider the semi-discretized problem (4.1) discretized this time over 10 nested dyadic grids with $N=2^{2 \times 10}=1048576=1024^{2}$ cells on the finest grid $J=10$.

In order to take into account the memory requirements of each resolution strategy, we estimate the array size of the working space needed by Radau 5 and ROCK4:

1. Radau5: $L_{1}=4 \times W_{1} \times W_{1}+12 \times W_{1}+20$ (from [23]);

2. ROCK4: $L_{2}=8 \times W_{2}($ from [1]);

where $W_{1}$ and $W_{2}$ are the number of unknowns solved by Radau 5 and ROCK4. In the case of a uniform mesh, the total number of unknowns is $W=3 \times 1024^{2} \approx 3.15 \times 10^{6}$ and thus, the global size $L$ required for each solver is:

1. Quasi-exact with Radau5: $W_{1}=W \approx 3.15 \times 10^{6}$ and $L=L_{1} \approx 4 \times 10^{13}$.

2. Splitting: $W_{1}=3, W_{2}=W \approx 3.15 \times 10^{6}$ and $L=L_{1}+L_{2} \approx 2.5 \times 10^{7}$.

3. MR/splitting with $\varepsilon=10^{-2}$ : $W_{1}=3, W_{2}=0.09 \times W \approx 2.9 \times 10^{5}$ and $L=L_{1}+L_{2} \approx 2.3 \times 10^{6} ;$ with an average data compression of $91 \%$.

Considering a standard platform on which each double precision value is represented by 64 bits, we shall require $2.3 \mathrm{~Pb}, 1.5 \mathrm{~Gb}$ and $140.4 \mathrm{Mb}$ respectively, for each solver. For standard computational resources, an implicit resolution with Radau5 is completely out of reach. These expensive memory requirements are strongly reduced with a splitting strategy but further reductions are achieved by adding a multiresolution adaptive procedure.

Figure 4.6 (top) shows the spatial representation of variable $a$ on the finest level corresponding to a $1024^{2}$ spatial discretization of problem (4.1) at an intermediary time $t=2$ and after one revolution at final time $t=4$. The corresponding data compressions $D C$ are respectively of $92.3 \%$ and $89.9 \%$, while the steepest spatial gradients of the front are always solved within the finest region taking into account that the splitting time step $\Delta t=4 / 1024$ remains bounded by $\Delta t_{\max }=4.2 \times 10^{-3}$ for $\Delta x=\Delta y=1 / 1024$ according to (3.6). For this case, six levels of grid discretization were used $^{3}$ from 5 to the finest grid 10 .

Bottom of Figure 4.6 shows the corresponding reaction time steps averaged within $\Delta t / 2$ for each point. We have the same distribution as in the previous $256^{2}$ case with reaction time steps going from $\Delta t / 2$ to time steps almost 22 times smaller depending of the local chemical activity. On the other hand, the spectral radius $\rho(\mathbf{B})$ estimated by ROCK4 is larger because of the finer spatial discretization of the Laplacian operator

\footnotetext{
${ }^{3}$ Same performance is obtained by considering 16 roots in each direction and $J=6$ levels of discretization.
} 
and it is of the order of 23000 , so if we consider a diffusion time step $\Delta t_{D}$ equal to the splitting time step, $s=16$ stages will be needed according to $(2.7)^{4}$. However, for a given tolerance of $\eta_{R O C K 4}=10^{-5}$, an initial time step given by $\Delta t_{D}=\Delta t$ is rejected to finally reach a relatively constant value of $2.5-3.5 \times 10^{-4}$ for which 5 stages instead of 16 are enough to guarantee the stability of the method. Finally, $11-15$ diffusion time steps $\Delta t_{D}$ are computed inside each splitting time step $\Delta t$.
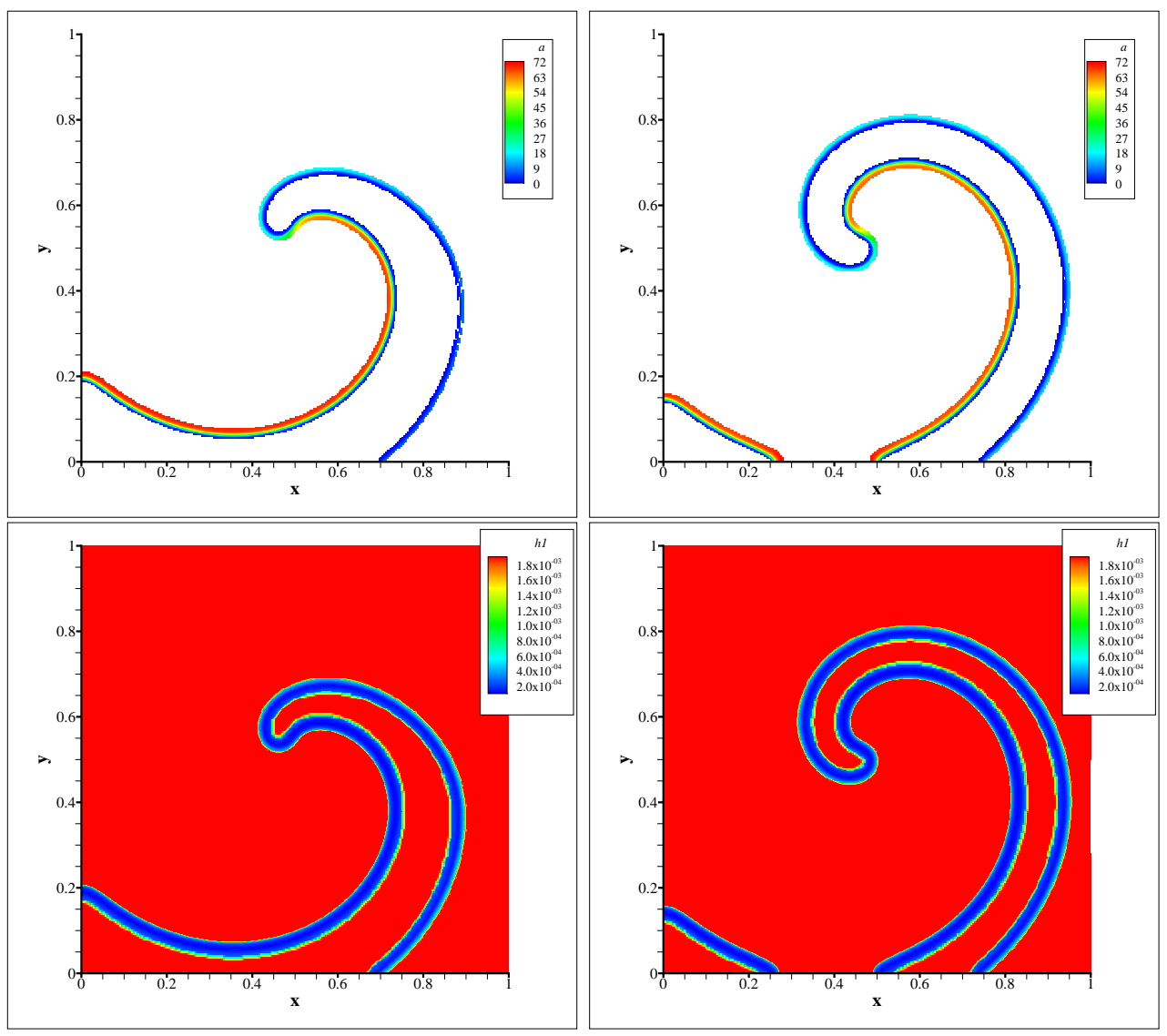

FIG. 4.6. 2D BZ spiral waves. Variable a on the finest grid (top) and local reaction time steps $h 1$ (bottom) at $t=2$ (left) and $t=4$ (right) with $\Delta t=4 / 1024$ and $\varepsilon=10^{-2}$. Finest grid: $1024^{2}$.

Figure 4.7 (top left) shows the adaptive splitting time steps obtained by the local error estimate procedure introduced in [11] with an accuracy tolerance of $\eta_{\Delta t}=$ $10^{-3}$. This is shown to be almost equivalent to the constant splitting time step $\Delta t=4 / 1024$ as in the previous $256^{2}$ spatial discretization case, where this splitting error is measured for a given semi-discretized problem. In general and for this kind of propagating phenomena, the adaptive scheme can be used to initially compute the corresponding constant splitting time step for a given accuracy.

In all these illustrations, the finest grid of computation is previously settled and

\footnotetext{
${ }^{4}$ It is important to notice that ROCK4 needs to save only 8 arrays of the size of the number of unknowns regardless the number of stages. One of these arrays contains the approximate solution used to estimate the local error in order to adapt the time step of integration within the prescribed tolerance.
} 
it is basically limited by the computational resources so that the multiresolution error is indicating the numerical approximation of the compressed spatial representation with respect to the semi-discretized problem regardless its spatial discretization as it is shown in Figure 4.7 (top right). The quasi-exact solution was computed with ROCK 4 and $\eta_{R O C K 4}=10^{-10}$ and took over $65072 \mathrm{~s}$ compared with $13943 \mathrm{~s}$ and $9529 \mathrm{~s}$ respectively for the splitting solution and the $\mathrm{MR} /$ splitting solution with $\varepsilon=10^{-2}$.
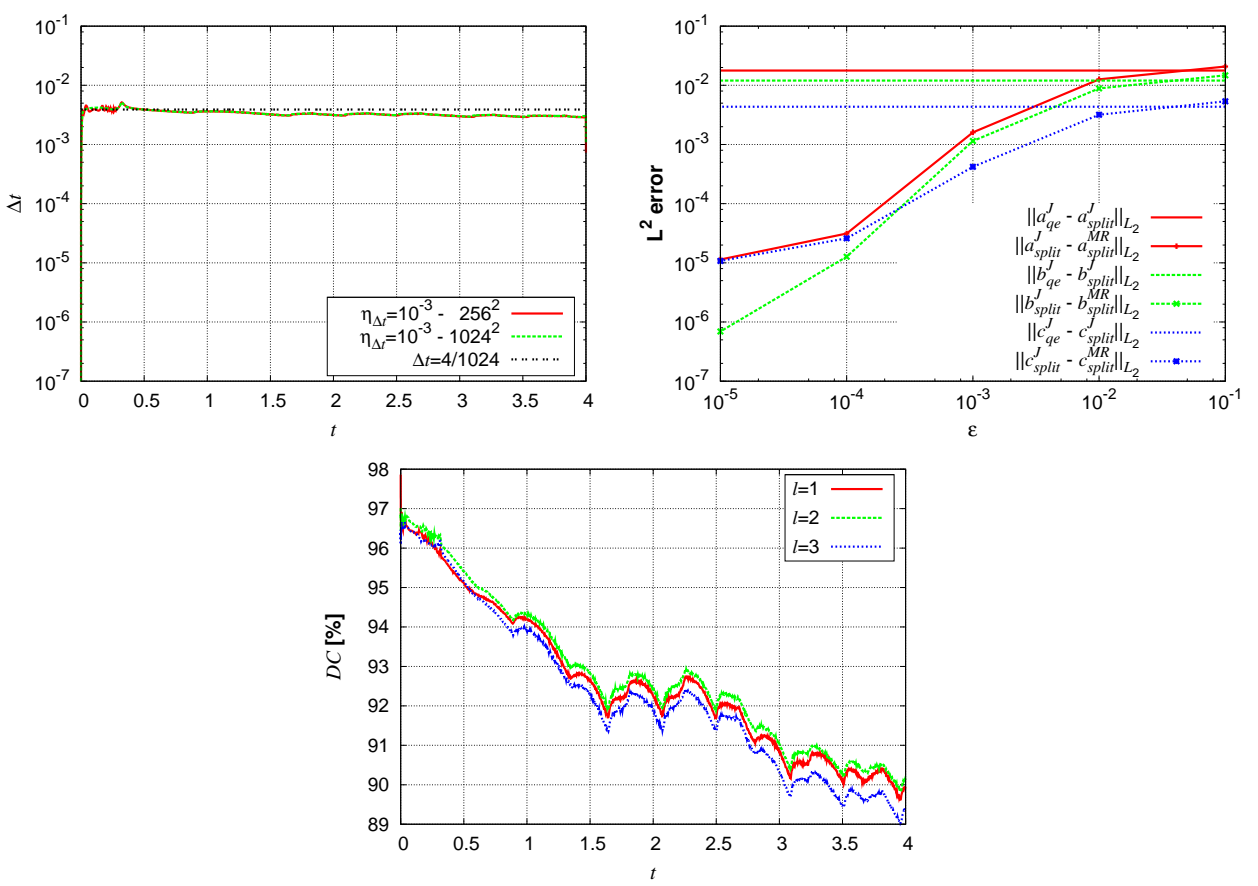

FIG. 4.7. 2D BZ spiral waves. Top left: time evolution of splitting time steps with $\eta_{\Delta t}=$ $10^{-3}$ and finest grids of $256^{2}$ and $1024^{2}$, with $\Delta t=4 / 1024$. Top right: normalized $L^{2}$ errors at $t^{\star}=4$ given by splitting technique on a uniform grid according to (3.3), and MR procedure according to (3.5) for several threshold values $\varepsilon$, splitting time step $\Delta t=4 / 1024$ and a finest grid of $1024^{2}$. Bottom: time evolution of data compression DC for prediction operators given by polynomial interpolations of different orders $2 l$ and a finest grid of $1024^{2}$.

Figure 4.7 (bottom) shows the dependence of the data compression on the definition of the prediction operator (2.11) according to $\S 3.1$. For higher order polynomial interpolations that yield more accurate and thus more compressed multiresolution representations according to (2.12), larger stencils are also needed so that the resulting data compression results of both opposite actions. In a general case, this is a problem dependent feature that can be a useful parameter to improve the performance of a multiresolution technique. A more detailed study of this dependence is out of the scope of the present work.

4.3. 3D BZ Equation. We consider now problem (4.1) in a $3 \mathrm{D}$ configuration with the same parameters considered in the $2 \mathrm{D}$ case for a time domain of $[0,2]$ and in a space region $[0,1]^{3}$. Initialization is made in the same way but with the coordinate angle $\theta$ given by

$$
\theta=\arctan \left(\frac{(x-0.5) \sin (\pi z)+(y-0.5) \cos (\pi z)}{(x-0.5) \cos (\pi z)-(y-0.5) \sin (\pi z)}\right)
$$


$z$ equal to zero corresponds to the previous $2 \mathrm{D}$ case. First, we take into account 8 nested dyadic grids with $N=2^{3 \times 8}=16777216=256^{3}$ cells on the finest grid $J=8$. Then, with a threshold value of $\varepsilon=10^{-2}$ and a splitting time step $\Delta t=4 / 1024$, the proposed numerical strategy features data compressions of $92.61 \%$ for the initial condition, $85.64 \%$ at $t=1$ when the scroll waves are fully developed and $81.42 \%$ at final time $t=2$. Figure 4.8 shows the scroll waves for variable $a$ at two different times and the adapted grid at $t=2$. The finest regions correspond to the neighborhood of the wavefront. The adaptive splitting time technique yields also a roughly constant splitting time step after a short initial transient whereas the choice $\Delta t=4 / 1024$ corresponds approximatively to $\eta_{\Delta t}=10^{-3}$. The CPU computation time was of about 41.94 hours with one processor.
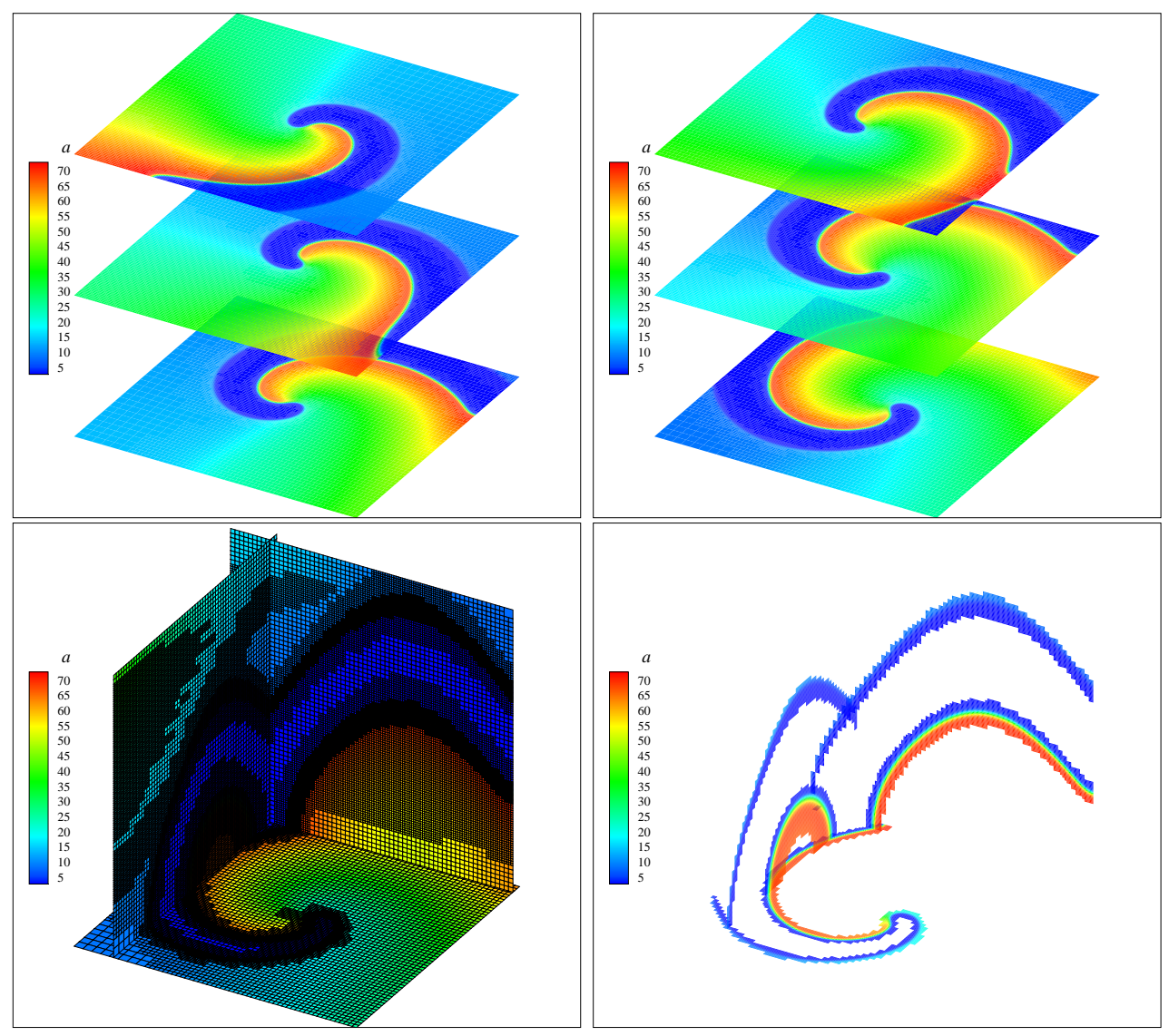

FIG. 4.8. $3 D$ BZ scroll wave. Top: evolution of variable a at $t=1$ (left) and $t=2$ (right). Bottom: Adapted grid (left) and finest grid (right) at $t=2$ for $\varepsilon=10^{-2}$. Finest grid: $256^{3}$.

In order to explore the feasibility and potential advantages of the method, let us consider 9 nested dyadic grids with $N=2^{3 \times 9}=134217728=512^{3}$ cells on the finest grid $J=9$. The initialization must take place on a intermediary grid, $j=8$ in this example. For this configuration, a two times larger splitting time step of $\Delta t=4 / 512 \approx 7.8 \times 10^{-3}$, and a threshold value of $\varepsilon=10^{-1}$ were chosen in order to have splitting and multiresolution errors potentially of the same order. Smaller threshold values yield larger simulation domains which are not longer feasible with 
the considered computing resource and the current state of development of the code. Figure 4.9 shows the adapted grid at $t=2$ and the corresponding finest regions. Compared with the $256^{3}$ case, finer regions are added at the steepest spatial gradients of the front. On the other hand, in order to globally guarantee (3.5), more refinement is needed at the lower levels according to (2.18) for a given threshold $\varepsilon$, where the multiresolution representation error (3.5) is always measured with respect to the corresponding uniform semi-discretized problem at the finest level. The latter is mainly limited by the computational resources and the desired level of accuracy of the spatial resolution. Data compressions are now of $95.79 \%$ for the initial condition, $91.56 \%$ at $t=1$ and $91.20 \%$ for final time $t=2$, with a CPU computation time of 159.4 hours.
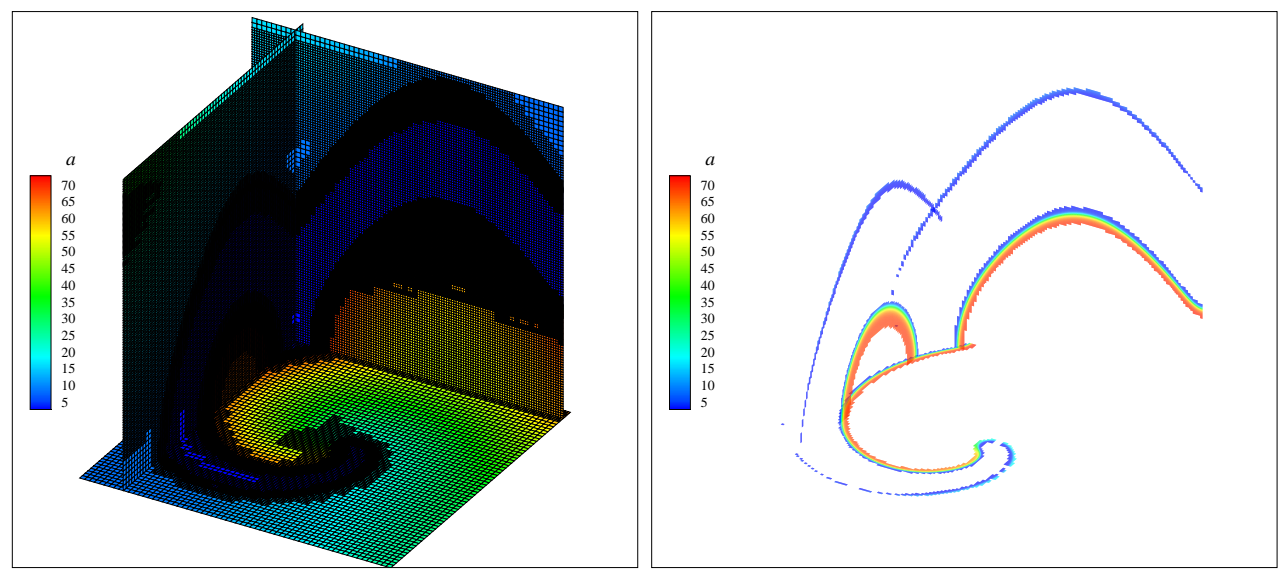

FIG. 4.9. 3D BZ scroll wave. Adapted grid (left) and finest grid (right) at $t=2$ for $\varepsilon=10^{-1}$. Finest grid: $512^{3}$.

Performing the same comparison concerning memory requirements, the total number of unknowns for this case is $W=3 \times 512 \times 512 \times 512 \approx 4.03 \times 10^{8}$ and the global size of $L$ required by each solver is:

1. Quasi-exact with Radau5: $W_{1}=W \approx 4.03 \times 10^{8}$ and $L=L_{1} \approx 6.5 \times 10^{17}$.

2. Splitting: $W_{1}=3, W_{2}=W \approx 4.03 \times 10^{8}$ and $L=L_{1}+L_{2} \approx 3.2 \times 10^{9}$.

3. MR/Splitting with $\varepsilon=10^{-1}: W_{1}=3, W_{2}=0.13 \times W \approx 5.3 \times 10^{7}$ and $L=L_{1}+L_{2} \approx 4.2 \times 10^{8}$; with a data compression of $87 \%$.

Therefore, we shall require at least $36.1 \mathrm{~Eb}, 190.7 \mathrm{~Gb}$ and $25.0 \mathrm{~Gb}$ of memory capacity, respectively for each solver.

5. Concluding Remarks and Outlook. Based on recent mathematical studies $[15,12]$ that allow to better characterize the behavior of splitting techniques for timespace stiff PDEs and in particular for splitting time steps much larger than the fastest scales present in the problem, this work introduces a new time operator splitting approach that exploits these results with an important gain of efficiency. Contrary to classical splitting strategies that consider splitting time steps set by the fastest time scales, in our strategy the splitting time step is chosen on the sole basis of the physics of the global phenomenon and its decoupling capabilities, but not related to any stability requirement of the numerical methods used to integrate each subsystem, even if strong stiffness is present. Dedicated high order time integration methods are then chosen for the reaction and diffusion problems to properly solve the entire spectrum of temporal scales associated with each independent problem. In this way, an effective decoupling 
of the time scale spectrum is achieved whenever this is allowed by the physics of the problem, so that different physical or numerical time scales associated with each problem can be isolated and treated independently by each numerical method.

We have shown that in the context of self-similar propagating waves a constant splitting time step is enough to capture the dynamics of the phenomenon. This was confirmed by an adaptive splitting strategy introduced in [11] that yields the same conclusion. Considering the adequate choice of higher order numerical methods with adaptive time stepping based on accuracy criteria such as Radau5 and ROCK4, the main error of the time integration is piloted by the splitting scheme and it is thus settled by the splitting time step even for stiff cases $[15,12]$. The latter is then computed based on error estimates of some physical feature such as the profile of the wavefront or its propagation speed. In a standard numerical strategy, this kind of preliminary study can be easily performed in 1D or smaller multidimensional configurations to then extrapolate to larger and more expensive computational domains taking into account that these splitting errors do not consider the spatial discretization error. However, from a practical point of view an adaptive splitting strategy can be used to initialize the splitting time step within the prescribed accuracy.

In order to improve the performance of the time integration strategy an adaptive mesh refinement technique based on spatial multiresolution was also implemented. One of the main advantages of such method is that for a given semi-discretized problem, the error introduced by the corresponding compressed spatial representation can be controlled. Even though a rigorous mathematical proof of multiresolution errors for parabolic problems is not yet available, the numerical results confirm the error estimates. In this way, for a problem represented with a spatial discretization limited mainly by the computational resources, the proposed MR/splitting strategy allows to track the corresponding numerical error of the simulation introduced by the numerical methods of resolution. As a consequence, the resulting highly compressed data representations as well as the accurate and feasible resolution of these stiff phenomena prove that large computational domains previously out of reach can be successfully simulated with conventional computing resources.

For the moment, we have focused our attention on reaction-diffusion systems in order to settle the foundations for simulation of more complex phenomena with fully convection-reaction-diffusion systems and more detailed models such as combustion with complex chemistry $[10,27]$. Therefore, an important amount of work is still in progress concerning programming features such as data structures, optimized routines and parallelization strategies for the time integration technique as well as for the multiresolution environment, even though the global CPU time is largely dominated by the time resolution for these stiff problems. For instance, some dedicated and efficient implementations have been recently developed for multiresolution applications $[3,4]$. Finally, when dealing with more complex systems such as complex or more detailed chemistry or stroke modeling in the brain, the source term involves many species (typically 50) and many reactions (typically several hundreds) or complex mechanisms. In such a case the integration of the source term leads to a heavy computational cost, even if it is embarrassingly parallel in the framework of operator splitting and if data compression issued from the multiresolution allows to improve its resolution as shown in [17]. Therefore, this field also requires some further studies in order to obtain high efficiency in terms of load balancing on parallel architectures. These issues constitute particular topics of our current research. 


\section{REFERENCES}

[1] A. AbDulle, Fourth order Chebyshev methods with recurrence relation, SIAM J. Sci. Comput., 23 (2002), pp. 2041-2054.

[2] B. L. Bihari and A. Harten, Multiresolution schemes for the numerical solution of 2-D conservation laws I, SIAM J. Sci. Comput., 18 (1997), pp. 315-354.

[3] K. Brix, R. Massjung, And A. Voss, A hash data structure for adaptive PDE-solvers based on discontinuous Galerkin discretizations, IGPM-Rep. 302, RWTH Aachen, (2009).

[4] K. Brix, S. Melian, S. Müller, And G. Schieffer, Parallelisation of multiscale-based grid adaptation using space-filling curves, ESAIM: Proc., 29 (2009), pp. 108-129.

[5] R. Bürger, R. Ruiz-BAier, K. Schneider, And M. SEPÚlveda, Fully adaptive multiresolution schemes for strongly degenerate parabolic equations in one space dimension, ESAIM: Math. Model. Numer. Anal., 42 (2008), pp. 535-563.

[6] J. C. Butcher, Implicit Runge-Kutta processes, Math. Comp., 18 (1964), pp. 50-64.

[7] A. Cohen, Wavelet Methods in Numerical Analysis, vol. 7, Elsevier, Amsterdam, 2000.

[8] A. Cohen, S.M. Kaber, S. Müller, and M. Postel, Fully adaptive multiresolution finite volume schemes for conservation laws, Math. Comp., 72 (2003), pp. 183-225.

[9] Y. D'Angelo, Analyse et Simulation Numérique de Phénomènes liés à la Combustion Supersonique, PhD thesis, Ecole Nationale des Ponts et Chaussées, 1994.

[10] M. S. DAY AND J. B. Bell, Numerical simulation of laminar reacting flows with complex chemistry, Combust. Theory Modelling, 4 (2000), pp. 535-556.

[11] S. Descombes, M. Duarte, T. Dumont, V. Louvet, and M. Massot, Adaptive time splitting method for multi-scale evolutionary PDEs., Confluentes Mathematici, (2011). Accepted for publication, available on HAL (http://hal.archives-ouvertes.fr/hal-00587036).

[12] S. Descombes, T. Dumont, V. Louvet, and M. Massot, On the local and global errors of splitting approximations of reaction-diffusion equations with high spatial gradients, Int. J. of Computer Mathematics, 84 (2007), pp. 749-765.

[13] S. Descombes, T. Dumont, V. Louvet, M. Massot, F. Laurent, and J. Beaulaurier, Operator splitting techniques for multi-scale reacting waves and application to low Mach number flames with complex chemistry: Theoretical and numerical aspects, In preparation, (2011).

[14] S. Descombes, T. Dumont, And M. Massot, Operator splitting for stiff nonlinear reactiondiffusion systems: Order reduction and application to spiral waves, in Patterns and waves (Saint Petersburg, 2002), AkademPrint, St. Petersburg, 2003, pp. 386-482.

[15] S. Descombes and M. Massot, Operator splitting for nonlinear reaction-diffusion systems with an entropic structure: Singular perturbation and order reduction, Numer. Math., 97 (2004), pp. 667-698.

[16] M. Duarte, Z. Bonaventura, M. Massot, A. Bourdon, S. Descombes, and T. Dumont, A new numerical strategy with space-time adaptivity and error control for multi-scale streamer discharge simulations., J. Comput. Phys., (2011). Accepted for publication, available on HAL (http://hal.archives-ouvertes.fr/hal-00573043).

[17] T. Dumont, M. Duarte, S. Descombes, M.A. Dronne, M. Massot, and V. Louvet, Simulation of human ischemic stroke in realistic $3 D$ geometry: A numerical strategy, Submitted to Bulletin of Math. Biology, (2011). Available on HAL (http://hal.archives-ouvertes.fr/hal00546223).

[18] B. EHLE, A-stable methods and Padé approximations to the exponential, SIAM J. Math. Anal., 4 (1973), pp. 671-680.

[19] I. R. Epstein And J. A. Pojman, An Introduction to Nonlinear Chemical Dynamics, Oxford University Press, 1998. Oscillations, Waves, Patterns and Chaos.

[20] R. J. Field, E. Koros, AND R. M. Noyes, Oscillations in chemical systems. II. Thorough analysis of temporal oscillation in the bromate-cerium-malonic acid system, J. Amer. Chem. Soc., 94 (1972), pp. 8649-8664.

[21] P. Gray and S. K. Scott, Chemical Oscillations and Instabilites, Oxford Univ. Press, 1994.

[22] E. Hairer, C. Lubich, and G. Wanner, Geometric Numerical Integration, Springer-Verlag, Berlin, 2nd ed., 2006. Structure-Preserving Algorithms for Odinary Differential Equations.

[23] E. Hairer And G. Wanner, Solving Ordinary Differential Equations II, Springer-Verlag, Berlin, 2nd ed., 1996. Stiff and differential-algebraic problems.

[24] A. HARTEN, Multiresolution algorithms for the numerical solution of hyperbolic conservation laws, Comm. Pure and Applied Math., 48 (1995), pp. 1305-1342.

[25] W. Jahnke, W. E. Skaggs, and A. T. Winfree, Chemical vortex dynamics in the BelousovZhabotinsky reaction and in the two-variable Oregonator model, J. Phys. Chem., 93 (1989), pp. $740-749$. 
[26] S. MülLER, Adaptive Multiscale Schemes for Conservation Laws, vol. 27, Springer-Verlag, 2003.

[27] H. N. Najm And O. M. KNIO, Modeling low Mach number reacting flow with detailed chemistry and transport, Journal of Scientific Computing, 25 (2005), pp. 263-287.

[28] O. Roussel, K. Schneider, A. Tsigulin, and H. Bockhorn, A conservative fully adaptive multiresolution algorithm for parabolic PDEs, J. Comput. Phys., 188 (2003), pp. 493-523.

[29] L. F. Shampine, B. P. Sommeijer, and J. G. Verwer, IRKC: An IMEX solver for stiff diffusion-reaction PDEs, J. Comput. Appl. Math., 196 (2006), pp. 485-497.

[30] B. SpORTisse, Contribution à la modélisation des écoulements réactifs: Réduction des modèles de cinétique chimique et simulation de la pollution atmosphérique, PhD thesis, Ecole Polytechnique, 1999.

[31] _ An analysis of operator splitting techniques in the stiff case, J. Comput. Phys., 161 (2000), pp. 140-168.

[32] G. Strang, Accurate partial difference methods. I. Linear Cauchy problems, Arch. Ration. Mech. Anal., 12 (1963), pp. 392-402.

[33] - On the construction and comparison of difference schemes, SIAM J. Numer. Anal., 5 (1968), pp. 506-517.

[34] J. G. VerWER, Explicit Runge-Kutta methods for parabolic partial differential equations, Appl. Numer. Math., 22 (1996), pp. 359-379.

[35] J. G. Verwer, B. P. Sommeijer, And W. Hundsdorfer, RKC time-stepping for advectiondiffusion-reaction problems, J. Comput. Phys., 201 (2004), pp. 61-79.

[36] J. G. Verwer And B. Sportisse, Note on operator splitting in a stiff linear case, Rep. MASR9830, (1998). 